\section{Bazyli Degórski, O.S.P.P.E.}

Pontificia Università S. Tommaso d'Aquino, Roma osppe.roma@gmail.com

ORCID: 0000-0003-1997-2769

DOI: http://dx.doi.org/10.12775/BPTh.2018.012

\section{Biblica}

et

Patristica

Thoruniensia

11 (2018) 2: 229-267

ISSN (print) 1689-5150

ISSN (online) 2450-7059

\title{
Il pasto per i poveri presso la Basilica Costantiniana di S. Pietro a Roma secondo la lettera di san Paolino di Nola a san Pammachio
}

\author{
The Meal to the Poor at the Constantine Basilica \\ of St Peter in Rome according to St Paulinus \\ od Nola's Letter to St Pammachius
}

\section{Uczta dla biedaków w Konstantyńskiej Bazylice św. Piotra w Rzymie na podstawie Listu św. Paulina z Noli do św. Pammachiusza}

Streszczenie. Artykuł analizuje fragment „Listu XIII” św. Paulina z Noli napisanego do św. Pammachiusza, który opisuje posiłek, jaki św. Pammachiusz zorganizował własnym sumptem dla biedaków Rzymu w czasie, gdy był senatorem i mnichem. Przed przystąpieniem do opisu posiłku, przedstawiliśmy najpierw autora listu, a następnie jego adresata. Analizowany fragment listu pozwala zauważyć, że dobroczynność, którą uskutecznia św. Pammachiusz, nie była tylko zwykłą filantropią, lecz opierała się na nauce Ewangelii i na obserwacji ówczesnego świata pogańskiego. Był to świat okrutny, który z zasady zmierzał do zaspokajania pożądań, zachcianek egoistycznych i hedonistycznych, pozbawionych altruizmu czy wyszych ideałów (nawet pogańskich!). W takim właśnie kontekście św. Paulin spostrzega posiłek przygotowany przez Pammachiusza jako prawdziwe ,igrzyska dla Kościoła” i przeciwstawia go okrutnym widowiskom pogańskim. Przedstawia on także Bazylikę św. Piotra, w której odbywał się ów posiłek, jako „teatr Chrystusa”, uwypuklając w ten sposób dodatkowo to przeciwstawienie. Analizowany fragment listu jest także ważny dla historyków sztuki i archeologów, gdyż dostarcza cennych szczegółów dotyczących wyglądu pierwszej Bazyliki św. Piotra na Watykanie, którą wybudował cesarz Konstanty Wielki. W samej bowiem rzeczy, opis potwierdza wspaniałość i wielkość tejże budowli, jakże ważnej dla chrześcijaństwa i ludzkości, która dotrwała do początku XVI wieku, kiedy to papież Juliusz II postanowił, by wznieść na jej miejscu obecną Bazylikę św. Piotra w Rzymie. 
Abstract. The articles analyzes a fragment of the "Letter 13", written by St Paulinus of Nola to St Pammachius. The shred describes the meal which St Pammachius offered at his own expenses - to the poor and needy people in Rome, when he was Senator and already a Monk.

The exact date of the occurrence is still debated, while it is ascertained what happened when St Pammachius was quite aged, namely when his wife, Therasia, was dead, and he used her properties and patrimony to help the needy people, willing to live a poor life as a monk.

The letter written by St Paulinus expresses his own appreciation for the behavior and initiative of the Senator-Monk Pammachius, whom he wanted to gratify with his esteem and appreciation.

In order to provide a better presentation of the Letter and its context, the present article introduces the two monks: the author of the Letter and the addressee. Both St Paulinus and St Pammachius had conducted a similar life, had many interests and concerns in common - though St Paulinus was 20 years older than St Pammachius - and were tied by bonds of spiritual friendship.

The fragment of the Letter analyzed in the present article clearly shows that the offering of the meal to the poor people by St Pammachius was not motivated by philanthropic attitude - in the secular sense of the term - but rather inspired by and rooted into the Gospel message, over and against the contemporary pagan mentality, quite often marked by self-centered and hedonistic desires and motivations and lacking sensitivity towards the others.

In this context, St Paulinus considers the meal offered by St Pammachius, as an authentic "competition in favor of the Church", as opposed to the pagan celebrations and St Peter's Basilica, where the meals took place, as "Christ's theatre".

The fragment of the Letter examined proves also of remarkable interest for historians and archeologists inasmuch as it provides important details about the ancient appearance of St Peter's Basilica. The description of the basilica, in fact, reports the splendor and the majesty of such key-place for all Christianity and humanity, as it appeared until the beginning of the $16^{\text {th }}$ century, when Pope Julius II replaced the ancient basilica with the new Vatican Basilica.

Słowa klucze: św. Paulin z Noli; św. Pammachiusz; Bazylika św. Piotra w Rzymie; Konstantyn Wielki; dobroczynność; archeologia; epistolografia.

Keywords: Saint Paulinus of Nola; Saint Pammachius; Basilica of St. Peter in the Vatican; Constantine the Great; Charity; archaeology; epistolography. 


\section{Introduzione}

L' articolo indende presentare al lettore un testo bello ed interessante tratto dalla "Lettera XIII" di san Paolino di Nola, indirizzata a san Pammachio. L'autore della lettera descrive e loda il pasto che Pammachio, monaco e senatore romano, organizzò a proprie spese per i poveri e i bisognosi della Città Eterna presso la basilica costantiniana di S. Pietro al Vaticano. Infatti, sin dall'inizio del cristianesimo, la beneficenza e la cura per i poveri costituì una delle attività principali e prioritarie della Chiesa e del movimento monastico sorto in seno ad essa

Paolino riportò grande impressione da questo pasto e la espresse nella sua "Lettera", ringraziandone il destinatario e la stessa bontà e generosità di tale gesto. Egli descrisse anche alcuni particolari concernenti la più antica Basilica di S. Pietro, costruita dall'imperatore Costantino il Grande. Queste notizie sono molto preziose per noi, perché della prima basilica di S. Pietro, fino ai nostri giorni, sono rimasti soltanto pochi frammenti.

Larticolo presenterà dapprima la figura dell'autore della "Lettera", ovverosia san Paolino di Nola, e in seguito quella del destinatario di essa: san Pammachio e, infine, passerà a trattare il brano della "Lettera" preso in esame.

\section{La figura di san Paolino di Nola}

San Paolino di Nola (il nome completo: Ponzio Meropio Anicio Paolino [Pontius Meropius Anicius Paulinus]) nacque a Bordeaux (Burdigala) in Gallia nel 355 (forse già nel 353) ${ }^{1}$, e morì a Nola in Campania il 22 giugno 431. Suo padre era il prefetto della Provincia dell'Aquitania e proveniva da un nobile e famoso casato di senatori e consoli romani. Paolino ebbe unottima istruzione, dato che fu discepolo del famoso poeta e retore pagano Ausonio ${ }^{2}$. Poco più che ven-

1 Cf. P. Fabre, St. Paulin de Nole et l'amitié chrétienne, 16.

2 Ausonio (Dec[i]mus Ausonius Magnus) nacque nel 310 circa a Bordeaux, e morì dopo il 393. Fu figlio di un medico di origine greca, Giulio Ausonio (Iulius Ausonius) che visse più o meno negli anni 290-378). Sua madre, Emilia Eonia (Aemilia Aeonia), fu figlia di Cecilio Argicio Arborio (Caecilius Argicius Arborius). Essa proveniva da ambo le parti dei genitori da famiglie aristocratiche della Gallia sud-occidentale. Ausonio studiò a Tolosa sotto la guida del fratello della madre, Arborio (Aemilius Magnus Arborius), il quale fu retore a Costantinopoli e precettore di uno dei figli dell'imperatore Costantino il Grande. Ausonio continuò gli studi nella città natia, a Bordeaux (Burdigala). Nel 334 circa, divenne grammatico e, in seguito, retore a Bordeaux. Nel 364 divenne precettore di Graziano, figlio 
tenne, nel 378, Paolino diventò senatore. In seguito, andò a Roma e fu nominato governatore della provincia della Campania. Si stabilì a Nola, ove possedette i suoi beni famigliari. Dopo la morte dell'imperatore Graziano, nel $383^{3}$, e dopo la salita al trono di Valentiniano $\mathrm{II}^{4}$, ariano che perseguitava non soltanto i cattolici, ma anche i sostenitori dell'usurpatore Massimo, Paolino, non benvoluto dal nuovo imperatore, ritornando in Aquitania si incontrò con il

di Valentiniano I, e ciò presso la sua corte imperiale a Treviri. Nel 370, divenne comes, e negli anni 375-378, in veste di questore del palazzo imperiale (Quaestor sacri palatii), dovette preparare delle nuove leggi. Nel 378, fu nominato prefetto dei pretoriani in Gallia. Nel 379, Ausonio fu console. Dopo la morte del suo alunno, Graziano, nel 383 ritornò a Bordeaux, ove visse sino alla morte, avvenuta dopo il 393. A proposito di Ausonio, cf. ad esempio: J. Demogeot, Etudes sur Ausone; C. Jullian, Ausone et Bordeaux; E.J. Kenney, W.V. Clausen, The Cambridge History of Classical Literature, 16-23; W.-L. Liebermann, P.L. Schmidt, D. Magnus Ausonius, 268-308; F.W. Bautz, Ausonius, Decimus Magnus, in BiographischBibliographisches Kirchenlexikon, 304-305; H. Sivan, Ausonius of Bordeaux. Genesis of a Gallic Aristocracy; A. Świderkówna (ed.), Słownik pisarzy antycznych, 106-109; A. Coşkun, Die gens Ausoniana an der Macht; B. Selter, Ausonius (Decimus Magnus Ausonius), 159-172; N. Rücker, Ausonius an Paulinus von Nola.

3 Graziano (Gratianus, Flavius Gratianus Augustus) nacque il 18 aprile 359 a Sirmio, e morì in Gallia, vicino a Lione (Lugdunum), il 25 agosto 383. Fu figlio dell'imperatore Valentiniano I e della sua prima moglie Severa. Nel 367, ottenne il titolo di Augusto, e nel 375, morto Valentiniano I, divenne imperatore della parte occidentale dell'impero. Nominò allora co-imperatore il suo fratellastro Valentiniano II che aveva appena quattro anni. A proposito di Graziano, cf.: O. Seeck, Gratianus 3, in Paulys Realencyclopädie der classischen Altertumswissenschaft, VII/2, 1831-1839; G. Gottlieb, Gratianus, in Reallexikon für Antike und Christentum, XII, 718-732; F.W. Bautz, Gratian, 289-290; P. Kehne, Gratian, 598-601.

4 Valentiniano II (Flavius Valentinianus) nacque nel 371, e morì tragicamente il 15 maggio 392 (la versione comune afferma che egli si suicidò, ma non è escluso che fu impiccato su comando del generale Arbogaste che de facto esercitava il potere). Valentiniano II fu figlio dell'imperatore Valentiniano I e della sua seconda moglie Giustina che fu ariana e perseguitava i cattolici. Valentiniano II regnò dal 375, anche se de facto il potere era esercitato da sua madre dirigendo l'Italia, l'Illiria e l'Africa settentrionale latina. Morto l'imperatore Graziano, nel 383 Magno Massimo (Magnus Maximus), il capo dell'esercito situato in Britannia, minacciò Milano, e nel 387 usurpò per se stesso il potere di essere il comandante delle province settentrionali dell'impero. Allora, Valentiniano II e sua madre, Giustina, fuggirono nella parte orientale dell'impero, presso Teodosio I (il Grande) che aveva in moglie la sorella di Valentiniano, Galla. Abitando in Oriente, Valentiniano II, sotto l'influsso dell'imperatore cattolico Teodosio, divenne cattolico. Teodosio, allora, lo aiutò e vinse nel 388 Magno Massimo. Ciò nonostante, dato che Valentiniano II aveva all'epoca soltanto dodici anni, fino alla sua maturità il potere fu esercitato, a nome suo, da Arbogaste, comandante romano di origine germanica. A proposito di Valentiniano II e dei suoi tempi, cf. ad esempio: W. Enßlin, Valentinianus II, 2205-2207; B. Croke, Arbogast and the Death of Valentinian II, 235-244; J.M. O'Flynn, Generalissimos of the western Roman empire; W.E. Roberts, Magnus Maximus: Portrait of a Usurper. 
vescovo di Milano sant'Ambrogio. Questi per primo gli spiegò i principi della fede cristiana. Non sappiamo se ciò sia avvenuto a Milano o altrove. Più tardi, Paolino mantenne con Ambrogio un contatto epistolare ${ }^{5}$. Paolino, nel 389, fu battezzato, però non da sant'Ambrogio, ma dal vescovo della sua città natia, san Delfino (Delphinus) ${ }^{6}$. Prima, negli anni 383-384, Paolino soggiornò in Spagna, ove si sposò con una cristiana molto esemplare, Terasia (Therasia) ${ }^{7}$, proveniente da una ricca famiglia che abitava a Barcellona. Dopo il battesimo, probabilmente per evitare la persecuzione da parte dell'imperatore ariano Valentiniano II, Paolino insieme alla moglie si trasferì in Spagna, nelle vicinanze dei Pirenei ${ }^{8}$. Ruppe allora i precedenti contatti (anche con il suo maestro, Ausonio) e decise di menare una vita cristiana più radicale. Per poter servire meglio i bisognosi, si privò delle ricchezze. Dedicava tanto tempo alla preghiera, allo studio della Sacra Scrittura e della teologia, nonché alla composizione di versi di stampo cristiano. Sotto l'influsso della morte del loro neonato e primogenito figlio, Celsuso ${ }^{9}$, ambo i genitori, incoraggiati da san Girolamo ${ }^{10}$,

5 Cf. Paulinus Nolanus, Epistula 3, 4; Ambrosius Mediolanensis, Epistula 58.

6 Le notizie su san Delfino, vescovo di Bordeaux, provengono dalla "Cronaca" di Sulpicio Severo, dalle cinque lettere scritte da san Paolino di Nola, da una nota di sant'Ambrogio, vescovo di Milano, e dagli Excerpta della Cronaca di Prospero di Aquitania. Delfino è il primo vescovo di Bordeaux storicamente documentato. Nel 380, egli partecipò al sinodo di Saragozza, convocato per esaminare la causa di Priscilliano, sospettato di eresia, e dei suoi seguaci. Per porre fine al priscillianesimo, san Delfino riunì a Bordeaux, nel 384, un sinodo al quale anche presiedette. Delfino fu un vescovo stimato ed influente e la sua fama superava i confini della diocesi. Egli mantenne la corrispondenza, fra gli altri, anche con sant'Ambrogio, vescovo di Milano. Durante il suo governo della diocesi, la fede cristiana si divulgò validamente. Ciò può essere testimoniato anche dal fatto che, a partire dal 400, i monumenti e le iscrizioni di quella regione posseggono quasi tutti delle scritte e dei simboli di stampo cristiano. Purtroppo, le opere e gli scritti di san Delfino non sono pervenuti fino ai nostri giorni. Delfino morì tra il 401 e il 403, e il suo successore divenne il presbitero Amando (Amandus), che fu anche amico di san Paolino di Nola. Al Martyrologium Romanum san Delfino fu iscritto sotto il 24 dicembre, ove leggiamo: "A Bordeaux in Aquitania, in Francia, san Delfino, vescovo, che fu unito a san Paolino da Nola da intima familiarità e si adoperò strenuamente per combattere l'eresia priscillianista". Attualmente, la diocesi di Bordeaux venera san Delfino il 30 dicembre. A proposito di san Delfino di Bordeaux, cf.: P. Fabre, Essai sur la chronologie de l'ouvre de s. Paulin de Nole, 57-65; idem, Paulin de Nole et l'amitié chrétienne, 252-261; E. Griffe, La Gaule chrétienne à l'époque romaine, I, 310-312; A. Mandouze (ed.), Storia dei santi e della santità cristiana, III, 269; H. Fros, F. Sowa, Ksiegga imion i świętych, 22; M. Maritano, Delfino di Bordeaux, 1354-1355.

7 Cf. Paulinus Nolanus, Carmen 21, 398-403.

8 Cf. Paulinus Nolanus, Carmen 21, 416 nn.; idem, Epistula 5, 4.

9 Cf. Paulinus Nolanus, Carmen 31.

10 Cf. Hieronymus, Epistula 58 [Ad Paulinum presbyterum]. 
decisero di rinunciare ai rapporti sessuali coniugali e di vivere alla stregua dei monaci.

A Barcellona, nel Natale del 393 o del 394, Paolino fu scelto presbitero dal popolo. Accettò di essere ordinato sotto la condizione di poter continuare la vita monastica. Lanno seguente, nel 395, si trasferì a Nola, ove possedeva ancora dei beni materiali. Insieme a Terasia, egli intese basarsi su una regola monastica. Lunica regola a lui accessibile furono gli statuti del monastero di Marmoutier, fondato da san Martino di Tours (Martinus Turonensis) ${ }^{11}$. Essi sono tramandati

11 San Martino di Tours nacque nel 317 o nel 335 a Sabaria in Pannonia, sul territorio dell'odierna Ungheria (attualmente la città si chiama Szombathely), e morì l'8 novembre 397 a Candes sul fiume Vienne nellodierna Francia. Suo padre, essendo tribuno di una legione romana, diede al figlio questo nome in onore del dio della guerra, Marte. Già durante l'infanzia, Martino si trasferi con i genitori a Pavia, ove il padre, ormai veterano militare, ottenne una proprietà terriera. In questa città Martino passò la fanciullezza. All'età di dieci anni, scappò da casa e rimase per due giorni dentro una chiesa (probabilmente a Pavia). Nel 331, l'imperatore emise un'ordinanza secondo la quale i figli dei veterani dovevano intraprendere il servizio di leva. Così, Martino fu inviato in Gallia nelle vicinanze di Amiens. Durante l'inverno del 335, egli incontrò un povero seminudo. Divise allora il suo ampio manto militare donandone una parte al medicante. La notte seguente, gli apparve in sogno Gesù vestito con la metà del manto di Martino. Quando Martino si svegliò, vide che il suo manto era integro. Il santo era, all'epoca, catecumeno, ma alla Pasqua ormai prossima ricevette il battesimo. Rimase soldato romano per circa vent'anni e raggiunse il grado di ufficiale, e ciò persino nelle cosiddette alae scholares (un corpo scelto). All'età di circa quarant'anni, decise di abbandonare il servizio militare. Tale decisione fu suggerita anche da un conflitto che egli abbe con il Cesare delle Gallie, Giuliano (quel Giuliano che nel 361 diventerà imperatore e passerà alla storia con l'appellativo di "Apostata"; cf. S. Trovato, Antieroe dai molti volti: Giuliano l'Apostata nel Medioevo bizantino, 127-134). Martino cominciò allora a combattere l'arianesimo. Nel 357, però, si trasferì sull'isola di Gallinara nellodierna provincia di Savona, ove per quattro anni condusse la vita eremitica. In seguito, si trasferì a Poitiers (Pictavium), nell'odierna Francia, e ormai in veste di Monaco, tutelato dal vescovo di Poitiers, sant'Ilario, fondò un monastero a Ligugé (uno dei più antici in Occidente). Nel 371, sulla richiesta degli abitanti di Tours, divenne vescovo di quella diocesi. In qualità di vescovo, san Martino continuò a condurre ancora la vita monastica, anche se unita ad unazione pastorale molto zelante. Fondò anche comunità monastiche. Nel 375, fondò un monastero situato vicino alle mura di Tours e, per un certo periodo, vi si trasferì. Il monastero si chiamava in latino Maius monasterium. Più tardi, sarà noto con il suo nome francese: Marmoutier. Come abbiamo detto, san Martino morì l'8 novembre 397 a Candes Saint Martin, ove si era recato per ripristinare la pace e la concordia tra il clero in lite. A proposito di san Martino, cf. le principali fonti: Sulpicius Severus, Vita S. Martini, CSEL 1, 107-137; idem, Epistula 1 [Ad Eusebium], CSEL 1, 138-141; idem, Epistula 2 [Ad Aurelium diaconum], CSEL 1, 142-145; idem, Epistula 3, CSEL 1, 146-151; idem, Dialogi, CSEL 1, 152-216. Cf. anche: P.J. Nowak (ed.), Sulpicjusz Sewer: Żywot świętego Marcina, 49-90; P.J. Nowak (ed.), Sulpicjusz Sewer: Listy, 91-108; P.J. Nowak (ed.), Sulpicjusz Sewer: Dialogi o życiu świętego Marcina, 
dall'amico e biografo di san Martino, Sulpicio Severo ${ }^{12}$, che descrive la vita dei monaci a Marmoutier. Imitandoli, Paolino vestì una tonaca di peli di cammello, speditagli da Sulpicio Severo ${ }^{13}$. I suoi capelli furono rasati, mangiava una sola volta al giorno, conformemente ai monaci orientali, cibandosi principalmente di pane e di ortaggi; beveva anche il vino, eccezion fatta per i giorni penitenziali (non dimentichiamo che Paolino era di Bordeaux!) ${ }^{14}$.

Paolino decise di conciliare la vita monastica claustrale con il servizio a favore dei poveri e dei bisognosi. Per tale scopo, costruì per loro a Cimitile, vicino a Nola, un rifugio. Nello stesso edificio, sopra le stanze per i bisognosi, si trovavano le celle dei monaci. Quindi, essi non avevano delle casette separate o capanne, alla stregua dei monaci di san Martino, bensì vivevano tutti insieme alla stregua dei cenobiti. La comunità monastica si riuniva la sera per celebrare i Vespri e, nella seconda metà della notte, recitava il Mattutino e le Lodi. San Paolino lavorava anche manualmente: coltivava un orticello. Ciò nonostante, attribuiva maggior importanza al lavoro intellettuale e allo studio ${ }^{15}$. Inoltre, seguendo i consigli di san Girolamo ${ }^{16}$, egli stesso insegnava ai suoi monaci quanto riguardava la Sacra Scrittura e la fede cattolica ${ }^{17}$.

Anche se il suo monastero seguiva naturalmente le leggi della povertà, san Paolino fece costruire una suntuosa basilica in onore di san Felice ${ }^{18}$.

109-190. A proposito degli studi su san Martino, cf. ad esempio: Société des Bollandistes (ed.), Bibliotheca Hagiographica Latina, 823-830; J. Fontaine, Vérité et fiction dans la chronologie de la "Vita Martini", 189-236; W. Nigg, Martin de Tours; C. Stancliffe, St. Martin and his hagiographer; G.-M. Oury, Saint Martin de Tours: L’homme au manteau partagé; A. Breukelaar, Martinus von Tours, 949-955; M. Starowieyski, Wstęp, 11-39; J. Drumm (ed.), Martin von Tours. Der Lebensbericht von Sulpicius Severus; J. Fontaine, Martin, hl., 1427-1428; M. Heinzelmann, Martin von Tours, in Reallexikon der Germanischen Altertumskunde, 365-369; R. Mensing, Martin von Tours; M. Happ, Alte und neue Bilder vom Heiligen Martin; A. Drouve, Der heilige Martin. Patron der Armen - Vorbild der Nächstenliebe.

12 Cf. B. Degórski, Il monachesimo latino nel Mediterraneo (secc. IV-VI), 117-118.

13 Cf. Paulinus Nolanus, Epistula 29, 1-2.

14 Cf. I. Gobry, Storia del monachesimo, 467.

15 Cf. I. Gobry, 467.

16 Cf. Hieronymus, Epistula, 53.

17 Cf. I. Gobry, 468.

18 Cf. ibidem. Ai tempi dei romani, sul territorio dell'odierna Cimitile si trovava un cimitero nel quale fu anche sepolto san Felice. Sopra la sua tomba fu edificato un mausoleo in forma di quadrato. Da ciò proviene l'antico nome della località, Cimiterium, che poi passò alla forma italiana moderna di "Cimitile". Quest'edificio antico sacrale, però, ben presto risultò troppo piccolo per contenere i numerosi pellegrini che volevano pregare sulla tomba di san Felice. Per tale ragione, nei primi anni del secolo V, san Paolino iniziò a costruire una nuova e più imponente chiesa in onore di san Felice. Questa costruzione aveva tre navate 
San Paolino stava anche in costante contatto con la Chiesa, scambiando lettere con i diversi suoi rappresentanti più significativi: sant'Agostino d'Ippona o san Girolamo ${ }^{19}$. Tra i suoi amici risulta anche Rufino di Aquileia, padre spirituale di Melania l’Anziana, insieme alla quale fondò dei monasteri latini a Gerusalemme ${ }^{20}$.

e terminava con unabside con tre nicchie. Inoltre, veniva abbellita con marmi preziosi ed affreschi. La vecchia e la nuova chiesa erano collegate con un atrio comune nel quale si trovavano alcune fontane.

19 Cf. ad esempio: Paulinus Nolanus, Epistula 1; Hieronymus, Epistula 53; idem, Epistula 58; idem, Epistula 85.

20 Cf. I. Gobry, 468. Melania l'Anziana (Senior) fondò il più antico monastero latino in Terra Santa. Ella fu figlia del console Marcellino (Marcellinus), fu aristocratica proveniente dalla famosa gens Antonia. Sposò l'ex-prefetto di Roma, Valerio Massimo (Valerius Maxi$m u s$ ). All'età di ventidue anni, perse sia il marito che due suoi figli. Decise allora di consacrarsi alla vita monastica. Affidò alla custodia di un tutore l'unico suo figlio a lei rimasto e nel 372, insieme ad altre aristocratiche romane partì alla volta di Alessandria. Lì conobbe Rufino di Aquileia (Tyrannius Rufinus) che divenne suo padre spirituale. Rufino, insieme con Girolamo, in precedenza apparteneva alla comunità monastica di Aquileia. Ebbe un’ottima preparazione monastica nella patria dei monaci, cioè in Egitto. Ciò nonostante, a causa della salute cagionevole, non osò mai imitare del tutto la severa vita dei primi monaci egiziani. A partire dal 372, Rufino abitò per sei anni ad Alessandria in Egitto incontrandosi con i più noti monaci egiziani e partecipando alle lezioni di Didimo il Cieco. Essendo uno zelante sostenitore della fede nicena, fu arrestato dagli ariani, ma grazie all'aiuto finanziario e della posizione sociale di Melania l'Anziana, riuscì a liberarsi dai persecutori (cf. Palladius, Historia Lausiaca 46, 3-4). Nello stesso tempo, Melania l'Anziana andò in Palestina e si stabilì a Gerusalemme, ove visse per ventisette anni. Fondò sul Monte degli Ulivi un monastero femminile latino, presso il quale costruì una casa per i pellegrini (cf. Palladius, Historia Lausiaca 46, 6). Quando nel 380 Rufino tornò a Gerusalemme, Melania fece costruire, accando al suo monastero, anche un monastero maschile, e Rufino ne divenne superiore. Egli divenne anche padre spirituale di Melania e delle sue monache. Questo compito, senza dubbio, doveva essere difficile ed impegnativo, perché nel monastero vivevano circa cinquanta monache (cf. Palladius, Historia Lausiaca 46, 5). La vita monastica in ambo i monasteri consisteva nel celebrare la liturgia, pregare, mortificarsi e nel lavorare (specialmente lavoro caritativo per i pellegrini, ma anche intellettuale: lo studio, la copiatura dei manoscritti, le traduzioni dal greco in latino delle opere di Origene). Oltre a tale attività, Rufino e Melania si prodigarono per l'unità della Chiesa ottenendo, tra l'altro, il cessare dello scisma provocato dell'ultra-cattolico e fanatico vescovo Paolino di Antiochia; condussero anche un efficace dialogo con gli pneumatomachi (cf. Palladius, Historia Lausiaca 46, 6). Purtroppo, Melania e Rufino, entusiasti del genio di Origene, divennero oggetto degli attacchi degli avversari del Grande Alessandrino, il che turbò assai la loro quiete monastica. La situazione si fece così difficile che la loro ulteriore permanenza a Gerusalemme divenne impossibile. Per tale ragione, circa il 399-400, Melania ritornò in Italia e andò a trovare suo cugino Paolino di Nola (cf. B. Degórski, Najstarszy monastycyzm żeński, 24). Rufino, invece, anch'egli verso il 400, si trasferì ad Aquileia e scrisse due "Apologie" difendendosi dalle accuse di condividere 
Paolino lo considerava uno dei più grandi conoscitori del monachesimo orientale ${ }^{21}$.

Quando nel 409 morì Paolo, amico di Paolino e vescovo di Nola, il nostro Santo divenne suo successore ${ }^{22}$. Esercitando il ministero episcopale, Paolino riuscì a mantenere lo stile monastico di vita. Con ciò egli imitò, senza dubbio, san Martino di Tours. Durante l'invasione di Alarico I nel $410^{23}$, trattando con l'invasore, san Paolino riuscì ad ottenere la liberazione dei propri fedeli ${ }^{24}$.

le tesi temerarie di Origene. Rufino continuò anche a tradurre in latino le opere dei Padri greci. A causa dell'avanzare nel 408 dei Visigoti guidati da Alarico, Rufino lasciò Aquileia e si rifugiò dapprima a Roma e poi nel monastero di Pinetum vicino a Terracina nel Lazio (cf. Rufinus, Prologus in Regulam Sancti Basilii). Nel 410, subito dopo il sacco di Roma, perpetrato da Alarico, Rufino, insieme a qualche amico, si rifugiò in Sicilia, ove morì con molta probabilità nei primi mesi del 411. A proposito di Rufino di Aquileia e di Melania l'Anziana, cf. ad esempio: H. Chisholm, Rufinus, Tyrannius, 820-821; F.X. Murphy, Rufinus od Aquileia; idem, Melania the Elder: a Biographical Note, 59-77; C.P. Hammond, The Last Ten Years of Rufinus' Life, 372-429; A.D. Booth, Quelques dates hagiographiques, 144-151; P. Caroline, H. Bammel, Der Römerbrieftext des Rufin und seine Origenes-Übersetzung; G. Fedalto, Rufino di Concordia (345 s. 410-411): tra Oriente e Occidente, Roma 1990; B. Flusin, Melania die Ältere, 490; H.R. Drobner, Rufinus, Tyrannius, 959-972; E. Bodart, Rufinus von Aquileia, 1088-1089; F. Drączkowski, Patrologia, 148-149 (a proposito di Rufino); M. Sgarbossa, I Santi e i Beati della Chiesa d'Occidente e d'Oriente, 326 (a proposito di Melania); G. Fedalto, Rufino di Concordia tra Oriente e Occidente; A. Pollastri, Melania senior, 3187-3188; J. Gribomont, Rufino di Aquileia, 4612-4614.

21 Cf. Paulinus Nolanus, Epistula 28, 5.

22 Nell'indirizzo dell'Epistula 149 (cf. anche l'Epistula 186), scritta da sant'Agostino d'Ippona negli anni 414-415, san Paolino di Nola viene chiamato coepiscopus. Gli anni 412-413, invece, vengono suggeriti dalla frase agostiniana del De Civitate Dei (cf. 1, 10, CCL 47, 11), ove il Vescovo d'Ippona, ricordando la preghiera fatta durante la distruzione della città da parte dei Goti nel 410, dice: "Paulinus noster, Nolensis episcopus". Allora, san Paolino divenne vescovo prima del 410, ma senza dubbio dopo il 408 (cf. Augustinus, Epistula 95, scritta nel 408, nella quale a proposito di san Paolino viene usato il titolo di episcopus).

23 Circa Alarico I (Flavius Alaricus) cf. ad esempio: O. Seeck, Alaricus 2, 1286-1291; P. Courcelle, Histoire littéraire des grandes invasions germaniques, 31-77; E. Demougeot, La formation de l'Europe et les invasions barbares, 2, De l'avènement de Dioclétien (284) à l'occupation germanique de l'Empire Romain d'Occident (debut du VI siècle), 451-464; G. Albert, Goten in Konstantinopel: Untersuchungen zur oströmischen Geschichte um das Jahr 400 n.Chr.; P.J. Heather, Goths and Romans. 332-489; B. Luiselli, Storia culturale dei rapporti tra mondo romano e mondo germanico, 384, 419-421, 556-557; L. Gatto, Storia di Roma nel Medioevo, 56-64; H. Wolfram, Die Goten. Von den Anfängen bis zur Mitte des 6. Jahrhunderts, 145-179; H. Sivan, Alaricus Rex. Legitimizing a Gothic King, 109-121; G. Pilara, Alarico I, 172-173; F.M. Ausbüttel, Germanische Herrscher, 73-87; Ph. Matyszak, Wrogowie Rzymu, 219-231; M. Meier, Alarich und die Eroberung Roms im Jahr 410. Der Beginn der "Völkerwanderung", 45-62; M. Meier, S. Patzold, August 410 - Ein Kampf um Rom. 
Negli anni 409-414, mori la moglie di Paolino, Terasia, ed egli pure si spense a Nola il 22 giugno $431^{25}$.

San Paolino oggi è noto specialmente come poeta cristiano ed autore di lettere. La maggior parte di questi scritti nacque durante il suo soggiorno a Cimitile, ovverosia fino agli anni 409-410 circa.

Le cinquantuno lettere, pubblicate da G. de Hartel ${ }^{26}$, sono indirizzate ad illustri personaggi di quel periodo, e tra gli altri: a Rufino di Aquileia ${ }^{27}$, a sant'Agostino d'Ippona $^{28}$, a Sulpicio Severo ${ }^{29}$, a Giovio (il principale negoziatore con

25 Cf. I. Gobry, 469; S. Costanza, C. Ricci, Paolino di Nola, 3826; B. Degórski, Il monachesimo latino, 118.

26 Cf. Paulinus Nolanus, Epistulae, CSEL 29 (cf. anche Augustinus Hipponensis, Epistulae, CSEL 34/1-2).

27 A Rufino san Paolino scrisse le seguenti lettere: Epistula 46, CSEL 29, 387-388; Epistula 47, CSEL 29, 388-389. A loro autenticità, però, viene messa in questione. Cf. A. Di Berardino, La poesia cristiana, 285.

28 A sant'Agostino Paolino indirizzò le seguenti lettere: Epistula 4, CSEL 29, 18-24; Epistula 6, CSEL 29, 39-42; Epistula 45, CSEL 29, 379-387; Epistula 50, CSEL 29, 404-423. In queste lettere il Vescovo di Nola chiede ad Agostino spiegazioni di alcuni brani della Bibbia, oppure di qualche questione riguardante la dottrina della Chiesa. Nel 401, sant'Agostino indirizzò, invece, a Pammachio una lettera (cf. Augustinus Hipponensis, Epistula 58), ringraziandolo di aver scritto ai Cristiani della Numidia, ove Pammachio aveva tanti possedimenti, invitandoli alla cessazione dello scisma donatista.

29 Paolino scrisse a Sulpicio Severo le seguenti lettere: Epistula 1, CSEL 29, 1-10; Epistula 5, CSEL 29, 24-39; Epistula 11, CSEL 29, 60-73; Epistula 17, CSEL 29, 125-128; Epistula 22, CSEL 29, 154-156; Epistula 23, CSEL 29, 157-201; Epistula 24, CSEL 29, 201-223; Epistula 27, CSEL 29, 238-240; Epistula 28, CSEL 29, 240-247; Epistula 29, CSEL 29, 247 -262; Epistula 30, CSEL 29, 262-267; Epistula 31, CSEL 29, 267-275; Epistula 32, CSEL 29, 275-301. Le lettere di san Paolino di Nola, indirizzate a Sulpicio Severo, costituiscono la parte più sostanziosa dell'epistolario di Paolino giunto ai nostri giorni. In queste lettere, il Vescovo di Nola ricorda alcuni eventi della propria vita (ad esempio: l'ordinazione presbiterale a Barcellona, o l'attesa prima di venire in Italia a Nola [cf. Epistula 1], oppure anche la freddezza che incontrò a Roma da parte del clero e persino di papa Siricio e la calorosa accoglienza da parte dei vescovi campani [cf. Epistula 5]). In altre lettere, Paolino parla del desiderio iniziale di Sulpicio Severo di trasferirsi stabilmente a Nola. Tale desiderio, però, poi cessò e Severo spedì al Vescovo di Nola solamente la sua, appena finita, Vita S. Martini. Altre lettere parlano dei monaci-corrieri che si mostrarono poco spirituali, oppure dell'invio di un cuoco, anche se pio, poco esperto nell'arte culinaria (cf. Epistula 23), dell'invio del mantello e della tunica (cf. Epistula 29), della richiesta, rivolta a san Paolino, affinché il Vescovo di Nola mandasse un proprio ritratto da collocare nel battistero, accanto al ritratto di san Martino (cf. Epistula 30), nonché del diniego di tale strana richiesta (cf. Epistula 32). Le lettere di san Paolino a Sulpicio Severo testimoniano anche di una profonda amicizia del Mittente, della sua severa vita ascetico-monastica, dellortodossia contraria agli errori pelagiani (cf. Epistulae 23-23), o addirittura degli stati mistici dell'Autore (cf. Epistula 28). San 
Alarico nel 410 $)^{30}$, a Desiderio ${ }^{31}$, a Pammachio ${ }^{32}$, a Delfino di Bordeaux ${ }^{33}$, ad Amando $^{34}$. Da questi scritti sprigiona una grande gioia, felicità che deriva

Paolino scrive anche dei pellegrinaggi in Terra Santa e del culto delle reliquie (cf. Epistulae 29 e 32 ).

30 A Giovio san Paolino scrisse soltanto una lettera (cf. Epistula 16, CSEL 29, 114, 125). Essa riguarda la Provvidenza e il Fato. Parla altresì, anche se superficialmente, dell'uso cristiano della letteratura pagana. Durante l'impero di Onorio, Giovio fu nominato da Stili-

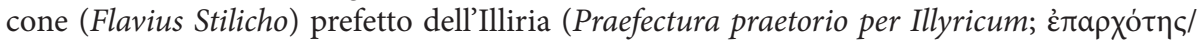

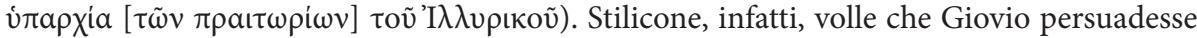
Alarico, affinché egli lo aiutasse nel sottomettere l'Illiria al potere di Onorio. In tal modo, Giovio contattò Alarico. Nel 408, morì tragicamente Stilicone, ucciso dal maestro degli uffici (magister officiorum), Olimpio (Olympius). Olimpio, invece, nel 409, cadde in disgrazia presso l'Imperatore. Così, nel 409, Giovio fu nominato patrizio (patricius) e prefetto del pretorio dell'Italia (praefectura praetorio Italiae, Illyrici et Africae). Nello stesso anno, Giovio dovette occuparsi del conflitto tra Onorio ed Alarico. Quell'ultimo, infatti, chiese che l'Imperatore riconoscesse i diritti del suo popolo. Giovio progettò delle trattative con Alarico a Rimini (Ariminum), suggerendo ad Onorio di accettare le richieste di Alarico. Ciò nonostante, l'Imperatore non lo volle fare $\mathrm{e}$, anzi, costrinse Giovio a giurare che non farà mai pace con Alarico. Neppure quando il Condottiero dei Goti restrinse le sue richieste, Giovio e gli altri ministri riuscirono a persuadere Onorio, affinché acconsentisse alle richieste di Alarico. Quest'ultimo, quindi, occupò Roma e mise sul trono Prisco Attalo (Priscus Attalus). Alla fine del 409, Giovio passò dalla parte di Alarico e di Prisco Attalo, i quali gli diedero il titolo di patrizio (patricius) e lo fecero prefetto del pretorio dell'Italia (praefectura praetorio Italiae, Illyrici et Africae). Desiderando un potere ancora maggiore, Giovio cercò di convincere Alarico, affinché abbandonasse Prisco Attalo. A proposito di Giovio, cf.: A.H.M. Jones, J.R. Martindale, J. Morris, The Prosopography of the Later Roman Empire, 623 (Iovius 3).

31 A Desiderio san Paolino spedì soltanto una lettera (cf. Epistula 43, CSEL 29, 363 $-369)$.

32 Al Monaco e senatore san Pammachio il Vescovo di Nola pure indirizzò una sola lettera (cf. Epistula 13, CSEL 29, 84-107), un cui frammento sarà analizzato proprio in quest'articolo.

33 Al vescovo san Delfino di Bordeaux san Paolino rivolse le seguenti lettere: Epistula 10, CSEL 29, 57-60; Epistula 14, CSEL 29, 107-110; Epistula 19, CSEL 29, 137-142; Epistula 20, CSEL 29, 142-149; Epistula 35, CSEL 29, 312-313. In queste lettere, egli esprime la sua gratitudine per il conferimento del battesimo e, inoltre, si scusa di non poter soddisfare la richiesta di Delfino di scriverre opere teologiche serie.

34 A sant'Amando, successore di san Delfino sulla sede episcopale di Bordeaux, san Paolino spedì le seguenti lettere: Epistula 2, CSEL 29, 10-13; Epistula 9, CSEL 29, 52-57; Epistula 12, CSEL 29, 73-84; Epistula 15, CSEL 29, 110-114; Epistula 21, CSEL 29, 149-154; Epistula 36, CSEL 29, 313-316. Non conosciamo esattamente le date della vita di sant'Amando. Egli morì probabilmente nel 432. Essendo ancora presbitero, preparò san Paolino al battesimo. Basandoci sulla testimonianza di Paolino e di san Gregorio di Tours, Amando era molto colto e pio. Non è escluso che Amando, al quale san Girolamo indirizzò la Epistula 55 riguardante diverse questioni esegetiche e una divorziata risposata, sia proprio il nostro 
dall'amicizia, e gratitudine per la possibilità di poter vivere, in comunione con gli altri, gli ideali ascetico-monastici ${ }^{35}$.

Per quanto riguarda la poesia di san Paolino di Nola, l'edizione di G. de Hartel conta trentatré carmina ${ }^{36}$. Tuttavia, soltanto ventotto di essi sono autentici. Infatti, i carmini I, IV, V, XXXII e XXXIII gli sono stati attribuiti erroreamente ${ }^{37}$. Quando Paolino era ancora pagano, ossia prima del 389 (l'anno in cui fu battezzato a Bordeaux dal vescovo san Delfino), le sue poesie avevano un contenuto laico. Dopo il battesimo, invece, la sua produzione poetica assunse un carattere profondamente religioso: nei carmini egli lodava Gesù Cristo, ma anche san Felice, di cui era particolarmente devoto. Dal momento del battesimo (dal 389) fino al 394, soggiornando ancora in Spagna, il futuro Vescovo di Nola compose i carmina VI-IX, e negli anni 393-394 i carmini X e XI, dedicandoli al suo maestro pagano, Ausonio. Il carmine XII fu scritto nel 395 in onore di san Felice. Stando ormai a Nola, nell'anno seguente (nel 396), scrisse un altro carmine (XIII) per il giorno "natalizio" (al cielo) di san Felice. Si tratta dei cosiddetti carmina natalicia (inni in occasione della nascita di qualcuno; in questo caso: della nascita al cielo). Da quel momento, per tale occasione, san Paolino ogni anno scriverà un carmen. Negli anni 397-408 o 409, Paolino scriverà ancora dodici di tali carmini in onore di san Felice, venerato il 14 gennaio (nel 397: il XIV; nel 398: il XV; nel 39938: il XVI; nel 400: il XVIII; nel 401: il XXIII; nel 402: il XXVI; nel 403: il XXVII; nel 404: il XXVIII; nel 405: il XIX; nel 406: il XX; nel 407: il XXI; nel 408 o nel 409: il XXIX $\left.{ }^{39}\right)^{40}$. Il carmen XVII fu scritto in onore del vescovo di Remesiana in Dacia, Niceta ${ }^{41}$, e il carmen XXIV del 400

sant'Amando. Amando, in veste di vescovo di Bordeaux, combatté vigorosamente il paganesimo e l'eresia priscilliana. A proposito di sant'Amando, cf.: D. de Sainte-Marthe, Gallia Christiana, 789-790; R. Étienne, Bordeaux antique; H. Crouzel, Les échanges littéraires entre Bordeaux et l'Orient au IV siècle, 316-319; H. Crouzel, L. Odrobina, Amando di Bordeaux, 225-226.

35 Cf. A. Swoboda, Pojęcie przyjaźni w listach św. Paulina z Noli i św. Sydoniusza Apolinarego: studium porównawcze; K. Ościłowski, Ideał chrześcijanina i jego realizacja: studium pism św. Paulina $z$ Noli.

36 Cf. Paulinus Nolanus, Carmina, CSEL 30, 1-3, 7-329.

37 Cf. S. Costanza, C. Ricci, Paolino di Nola, 3828.

38 Cf. L. Sciajno (ed.), Paolino di Nola. Il carme 15 (Natalicium IV).

39 Cf. A. Di Berardino, La poesia cristiana, 285-290; S. Costanza, C. Ricci, Paolino di Nola, 3828 .

40 A proposito di tutti i carmina natalicia, cf. la seguente dissertazione dottorale: R.B. Healy, The Carmina Natalicia of Paulinus of Nola.

41 Possediamo scarse notizie riguardanti la vita di Niceta. I frammenti delle sue opere a noi rimasti (cf. Clavis Patrum Latinorum 646-652) furono pubblicati in Patrologiae 
è una sorta di lunga e prolissa lettera a Citerio di Aquitania (Cytherius), a sua moglie e al loro figlio sacerdote novello. Negli anni 401-404, Paolino compose il carmen XXV. Si tratta di un epitalamio (epithalamium) composto per il matrimonio di Giuliano d'Eclano (il futuro vescovo di Eclano e pelagiano) ${ }^{42}$

Latinae cursus completus 52, 837-876; Patrologiae Latinae Supplementum 3, 189-202; A.E. Burn, Niceta of Remesiana. His Life and Works; C.H. Turner, De vigiliis (editio critica), 305-320; idem, De psalmodiae bono sive De utilitate credendi, 225-252; K. Gamber, Niceta von Remesiana. Instructio ad competentes. Niceta fu vescovo di Remesiana (lodierna Bela Palanka in Serbia) già nella seconda metà del IV secolo. Di Niceta di Remesiana, oltre a san Paolio di Nola, scrisse Gennadio di Marsiglia (cf. Gennadius Scholasticus seu Gennadius Massiliensis, De viris inlustribus 22) e Cassiodoro (cf. Cassiodorus, Institutiones divinarum et saecularium litterarum 16). Nel 414, anche papa Innocenzo I inviò a Niceta una lettera (cf. Innocentius I, Epistula 17). Il Papa lo menziona anche un po' prima, nel 409 circa. (cf. Innocentius I, Epistula 16). Niceta venne in Italia almeno due volte: nel 398 e nel 402. Morì dopo il 414, ma non possiamo esattamente stabilire la data del suo decesso. A proposito di Niceta, cf. anche: W.A. Patin, Niceta, Bischof von Remesiana als Schritsteller und Theologie; O. Bardenhewer, Geschichte der altkirchlichen Literatur, 598-605; É. Amann, Nicétas de Rémésiana, 477-479; J. Zeiller, Un ancien évêque d'Illyricum, peut-être auteur du "Te Deum", Saint Niceta de Remesiana, 356-369; D.M. Pippidi, Niceta di Remesiana e le origini del cristianesimo daco-romano, 99-117; G.C. Walsh, Writings of Niceta of Remesiana; K. Gamber, Ist Niceta von Remesiana der Verfasser von De Sacramentis?, 153-172; idem, Ist Niceta von Remesiana der Verfasser des pseudoambrosianischen Sermo De Spiritu Sancto?, 204-206; idem, Fragen zu Person und Werk des Bischof Niceta von Remesiana, 222-231; D.B. Saddington, The Educational Effect of Catechical Instruction in the Fourth Century A.D., 249-271; Z. Senjak, Niceta von Remesiana. Christliche Unterweisung und christliches Leben im spätantiken Dacien, Freiburg 1975; M.G. Mara, Ambrogio di Milano, Ambrosiaster e Niceta, 180-183; eadem, Niceta di Remesiana, A. Di Berardino (ed.), Dizionario patristico e di antichità cristiane, 2397-2398; C. Riggi, La figura di Niceta di Remesiana secondo la biografia di Gennadio, 189-200; idem, Niceta di Remesiana. Catechesi preparatorie al battesimo; B. Degórski, Katechezy przedchrzcielne Nicetasa z Remezjany, 107-111; H.C. Brennecke, Nicetas von Remesiana, 460-463; M. Zelzer, Die "Instructio ad competentes" des Nicetas von Remesiana und die Salzburger Slawenmission, , 321-327; M. Guire, Nicetas of Remesiana, 356; M.G. Mara, Niceta di Remesiana, A. Di Berardino (ed.), Nuovo dizionario patristico e di antichità cristiane, 3489-3490; B. Degórski, Il mistero dell'incarnazione nel commento di san Niceta di Remesiana al «Simbolo Apostolico», 231-237 Ph. Blasen, Nicetas of Remesiana - A Missionary Bishop in Dacia?, 39-49; A. Soroceanu, Niceta von Remisiana Seelsorge und Kirchenpolitik.

42 Giuliano fu figlio del vescovo di Eclano (Aeclanum, lodierna Mirabella Eclano in provincia di Avellino), Memorio (Memorius), e di Tizia (Titia), figlia di Emilio (Aemilius), vescovo di Benevento. Non sappiamo con precisione se sia nato ad Eclano. Infatti, alcuni studiosi sostengono che nacque nellodierna Ceglie Messapica in Puglia. Non conosciano neanche la data della sua nascita. Sappiamo soltanto che nel 408 fu ordinato diacono. Giuliano ottenne una profonda educazione ed aveva una brillante intelligenza. Anche per questi motivi, più o meno nel 411, papa Innocenzo I lo nominò vescovo di Eclano. Fu un pasto- 
e di sua moglie Tizia (Ticia; oppure: Ja). Il carmen XXXI, invece, fu stilato sotto l'influsso della morte del figlio neonato di san Paolino e di Terasia, Celso. Questo carmine è composto di 316 distichi elegiaci di grande valore letterario ${ }^{43}$. E difficile stabilire la data precisa della sua composizione, forse da collocarsi negli anni 393-408 oppure, con più probabilità, negli anni 393-401 ${ }^{44}$. In tutti i carmina (eccezione fatta per il carmine VI in onore di san Giovanni il Battista,

re zelante e buono e si occupò anche dei bisognosi: distribuì tutti i suoi beni materiali, e ciò per supplire alla povertà della popolazione locale causata dall'invasione del Vandali in Campania. Nel 418, papa Zosimo, con il documento Tractoria, scomunicò Giuliano a causa della condivisione degli errori dei pelagiani. Il Vescovo di Eclano, perciò, fu costretto ad andare in esilio, assieme a settanta altri vescovi pelagiani. Durante l'esilio, Giuliano fu ospitato da Teodoro, vescovo di Mopsuestia in Cilicia sul fiume Piramo (nellodierna Turchia) e da Nestorio, vescovo di Costantinopoli. Anche in esilio cercò di difendere le proprie tesi pelagiane divenendo il più influente di tale gruppo eterodosso. Non sappiamo ove sia morto: o in Oriente, o in Sicilia. Morì prima del 455. A proposito di Giuliano di Eclamo, cf.: A. Bruckner, Julian von Eclanum. Sein Leben und seine Lehre; A. Jülicher, Iulianos 12, in Paulys Realencyclopädie der classischen Altertumswissenschaft, 19-22; Y. de Montcheuil, La polémique de saint Augustine contre Julien d'Éclane d'après l'Opus imperfectum, 193-218; G. Bowman, Des Julian von Aeclanum Kommentar zu den Propheten Osee, Joel und Amos; H.I. Marrou, La canonization de Julien d'Éclane, 434-437; F. Refoulé, Julien d'Éclane, théologien et philosophe, 42-84, 233-247; F.-J. Thonnard, Laristotélisme de Julien d'Éclane et de Saint Augustin, 296-304; N. Cipriani, Aspetti letterari dell'Ad Florum di Giuliano d'Eclano, 125-167; Y.-M. Duval, Julien d'Éclane et Rufin d'Aquilée. Du concile de Rimini à la répression pélagienne, 243-271; N. Cipriani, Echi antiapollinaristici e aristotelismo nella polemica di Giuliano d'Eclano, 373-389; A. McGrath, Divine justice and divine equity in the controversy between Augustinus and Julian of Eclanum, 312-319; M. Lamberigts, Julian of Aeclanum: A Plea for a Good Creator, 5-24; J. Lössl, “Te Apulia genuit” (c. Iul. imp. 6.18). Some notes on the Birthplace of Julian of Eclanum, 223-239; L. Barclift, In Controversy with Saint Augustine: Julian of Aeclanum on the Nature of Sin, 5-20; Ch. Pietri, Prosopographie de l'Italie chrétienne (313-604), 1175-1186; J. Lössl, Julian von Aeclanum. Studien zu seinem Leben, seinem Werk, seiner Lehre und ihrer Überlieferung; M. Lamberigts, Recent Research into Pelagianism with Particular Emphasis on the Role of Julian of Aeclanum, 175-198; J. Lössl, Julian of Aeclanum on Pain, 203-243; M.C. Pennacchio, Lesegesi patristica di Osea nei secoli IV-V. Teodoro di Mopsuestia, Teodoreto di Ciro, Cirillo di Alessandria, Girolamo, Giuliano di Eclano; R. Villegas Marín, En polémica con Julián de Eclanum, 81-124; A. Nazzaro (ed.), Giuliano d'Eclano e l'Hirpinia Christiana. Atti del Convegno Internazionale di Studi; A.U. Sommer, Das Ende der antiken Anthropologie als Bewährungsfall kontextualistischer Philosophiegeschichtsschreibung: Julian von Eclanum und Augustin von Hippo, 1-28; V. Grossi, Giuliano d'Eclano, 2309-2311; B. Alexanderson, Quelques idées sur le texte et l'interprétation des cuvres de Julien d'Éclane Expositio libri Iob et Tractatus prophetarum Osee, Iohel et Amos, avec des remarques sur le texte et l'interprétation des commentaires de Jérôme.

43 Cf. A. Di Berardino, La poesia cristiana, 287.

44 Cf. S. Costanza, C. Ricci, Paolino di Nola, 3828. 
composto di 330 esametri, e dei carmini VII-IX che è una parafrasi dei Salmi 1, 2 e 136) san Paolino si serve dei generi letterali classici e noti, usati nella poesia:

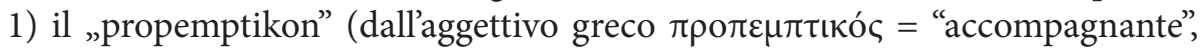
dal verbo $\pi \rho \circ \varepsilon \varepsilon \dot{\mu} \pi \omega$ = "accompagno"; tali poesie venivano composte quando qualcuno si recava in viaggio e si doveva esprimere, per tale ragione, il dolore della separazione e del distacco, oppure per augurargli un felice viaggio), 2) l'“epitalamio" (un inno nuziale scritto in occasione del matrimonio di qualcuno; dal gr. $\dot{\pi} \pi \dot{i}=$ "di; su", "sul tema" e 9á $\lambda \alpha \mu o \varsigma=$ "talamo"), 3) la consolazione (di qualcuno che è triste, mesto), o i già menzionati 4) carmina natalicia. Anche se il Vescovo di Nola si serve di questi generi letterari noti e classici, egli li arricchisce notevolmente, dando ad essi profondi contenuti cristiani.

\section{La persona di san Pammachio}

San Pammachio nacque nel 330 circa, e morì nel $410^{45}$. Apparteneva alla famosa famiglia aristocratica romana dei Furi (Gens Furia), i cui membri svolsero alti incarichi ed uffici durante tutto il periodo della Repubblica. Il primo dei Furi che ottenne l'incarico di console fu, nel 488 avanti Cristo, Sesto Furio Medullino Fuso (Sextus Furius Medullinus Fusus). Egli svolse tale ufficio insieme al console Spurio Nauzio Rutilo (Spurius Nautius Rutilus) ${ }^{46}$. Il più famoso, però, della famiglia dei Furi fu Marco Furio Camillo (Marcus Furius Camillus;446 ca. avanti Cristo - 365 avanti Cristo ${ }^{47}$, considerato il Secondo Fondatore di Roma,

45 Così brevemente descrive san Pammachio Palladius, Historia Lausiaca 62, 268-269:

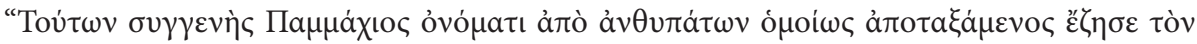

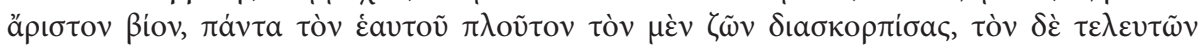

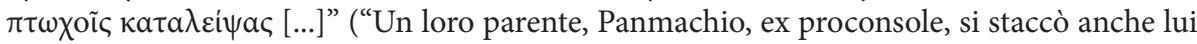
dal mondo e visse la più perfetta delle vite. Di tutta la sua ricchezza distribuì una parte mentre era in vita, e il resto lasciò ai poveri in atto di morte [...]").

46 Egli, per la prima volta, viene menzionato dallo storico e retore Dionigi di Alicarnasso (60 avanti Cristo - 7 dopo Cristo) nell'opera intitolata "Antichità Romane", mentre parla del 493 avanti Cristo, e poi menzionando la cosiddetta Secessio plebis del 494 avanti Cristo (cf. Dionysius Halicarnasseus, Antiquitates Romanarum 8, 16). Nel 488 avanti Cristo, fu eletto console insieme al già menzionato Sesto Furio Medullino Fuso. Ciò avvenne nell'anno in cui Gneo Marcio Coriolano (Gnaeus Marcius Coriolanus) guidò contro Roma l'esercito dei Volsci (cf. Titus Livius, Ab Urbe condita 2, 39).

47 A proposito di Marco Furio Camillo cf. la fonte principale: Titus Livius, Ab Urbe condita 5, 10; 6, 4; Plutarchus, Vitae parallelae. Camillus (3); Polybius, Historiae 2, 18; Eutropius, Breviarium ab Urbe condita 1, 20. Circa gli studi moderni che lo riguardano, cf.: Th. Mommsen, Römische Forschungen, II, 113-152; O. Hirschfeld, Zur Camillus-Legende (1895), in idem, Kleine Schriften, 273 nn.; E. Täubler, Camillus und Sulla, 219; E. Burck, 
insignito anche del più alto titolo di Pater Patriae ${ }^{48}$. Egli fu probabilmente il primo dei Romani che abbia ottenuto un tale titolo.

San Pammachio, in gioventù, studiò a Roma retorica insieme al suo amico

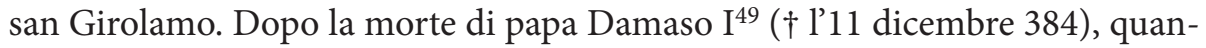
do Girolamo dovette lasciare Roma (più o meno nella primavera del 385), Pammachio sposò Paolina, la seconda figlia della matrona ed aristocratica romana santa Paola ${ }^{50}$, figlia spirituale diletta di san Girolamo.

Die Erzählungskunst des T. Livius, 109 nn.; J. Hubaux, Rome et Véies, recherches sur la chronologie légendaire du Moyen Age romain; A. Momigliano, Secondo contributo alla storia degli studi classici, 89 nn.; R.M. Ogilvie (ed.), A Commentary on Livy: Books 1-5, 669-670; J. Bayet, Appendice à l'édition de Tite Live, Histoire romaine, 93-170; J. Hellegouarc'h, Le principat de Camille, 112-132; J. Gagé, La Némésis de Camille, 111-138; G. Dumézil, Camillus: A Study of Indo-European Religion as Roman History; P. Traves, Camillo, Marco Furio, 367-368; P. Martin, L’idée de royauté à Rome, des origines au principat augustéen, 360-382; G. Dumézil, Mythe et épopée, 1163-1271 e 1288-1310; W. Eder, Furius [I 13], 715-716; U. Walter, Marcus Furius Camillus - die schattenhafte Lichtgestalt, 58-68; Ph. Akar, Camille et la Concorde, 205-215; U. Eigler, Camillus, 241-246.

48 A proposito del titolo di Pater patriae, cf.: A. Alföldi, Der Vater des Vaterlandes im römischen Denken; J.P.V.D. Balsdon, Pater patriae.

49 San Damaso I nacque nel 305 circa a Roma o a Guimarães nellodierno Portogallo, e morì a Roma l'11 dicembre 384. Il suo pontificato iniziò il $1^{\circ}$ ottobre 366 e durò sino alla sua morte. A proposito di san Damaso I, cf.: O. Marucchi, Il primato di papa Damaso nella storia della sua famiglia; A. Ferrua, Epigrammata Damasiana; F.X. Seppelt, Geschichte der Päpste von den Anfängen bis zur Mittel des zwanzigsten Jahrhunderts, I, 109-126; A. Lippold, Ursinus und Damasus, 105-128; M.H. Sheperd, The Liturgical Reform of Damasus, 847-863; M. Green, The Supporters of the Antipope Ursinus, 531-538; J. Taylor, St. Basil the Great and Pope Damasus, 183-203, 261-274; P. Nautin, Le premier échange épistulaire entre Jérôme et Damase: lettres réelles ou fictives?, 331-334; R.E. Reynolds, An early Medieval Mass Fantasy, 73-89; C. Carletti, Damaso I, 349-372; L. Grig, Portraits, Pontiffs and the Christianization of Fourth-Century Rome, 203-230; Ch. Pietri, M. Ghilardi, Damaso, 1325-1329; U. Reutter, Damasus, Bischof von Rom (366-384). Leben und Werk; M. Löx, Monumenta sanctorum. Rom und Mailand als Zentren des frühen Christentums; A. Aste (ed.), Gli epigrammi di papa Damaso I; D. Trout (ed.), Damasus of Rome: The Epigraphic Poetry.

50 A proposito di santa Paola e delle sue figlie, nonché del monachesimo femminile, iniziato tra le aristocratiche romane da san Girolamo, cf.: P. Antin, Le monachisme selon saint Jérôme, 71-118; A. Arjava, Jerome and women, 5-18; D. Balboni, Fabiola, in Bibliotheca Sanctorum, V, 431; A. Brunot, Sainte Paule et Sainte Eustochium, 259-264; E. Burke, St. Jerome as a Spiritual Director, in A Monument to St. Jerome, 145-169; F. Caraffa, Eustochio, 302-304; H. Cecchetti, "Hieronymus ab Aventino in Bethlehemiticum coenobium" da un libro di prossima pubblicazione, 4; B. Degórski, Opaci okresu Teodozjańskiego, 5-51 (special-

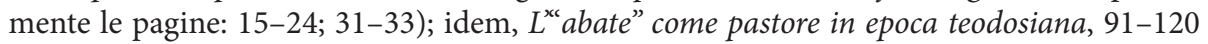
(in particolare pp.: 98-103; 107-109); idem, Korzenie duchowości paulińskiej. Pierwsi mnisi egipscy, 11-65 (specialmente le pagine: 49-62); idem, Najstarszy monastycyzm żeński, 5-59; 
Pammachio fu il primo senatore Romano divenuto monaco. Infatti, quando nel 397 sua moglie Paolina ${ }^{51}$ morì a causa del parto, Pammachio divenne monaco ${ }^{52}$ e cominciò una intensa attività caritativa. Fondò, tra l'altro, insieme a Fabiola di Roma ${ }^{53}$, una casa per poveri nel Porto di Roma (Por-

B. Degórski, Św. Hieronim. Listy do Eustochium. Listy 22, 31, 108 (passim); G. Del Ton, Paola di Roma, 123-136; G.D. Gordini, Forme di vita ascetica a Roma nel IV secolo, 7-57; idem, Origine e sviluppo del monachesimo a Roma, 220-260; idem, Il monachesimo romano in Palestina nel IV secolo, 85-107; idem, Marcella di Roma, 644-645; idem, Lopposizione al monachesimo a Roma nel IV secolo, 19-35; J. Górny, Udział wspólnot monastycznych w rozwoju życia intelektualnego i ascetycznego Kościoła na przełomie IV-V wieku wświetle pism św. Hieronima, 537-578; I. Grego, Betlemme, dolce patria mia!, 63-84; L. Gutierrez, El monaquismo romano y San Jerónimo, 49-78; idem, St Jerome and roman Monasticism. A historical Study on his spiritual Influence, 256-307; P. Laurence, Jérôme et l'ancilla Christi: servitude et liberté, 411-429; idem, Jerôme et le nouveau modèle féminin; idem, Les représentations de la "domina" chez Jérôme, 41-55; idem, Rome et Jérôme: des amours contrariées, 227-249; idem, Jérôme et les "puellae": un vocabulaire connoté, 1039-1063; idem, Ivresse et luxure féminines: les sources de Jérôme, 885-899; idem, Jérôme, la culture grecques et les femmes, 315-331; idem, L'implication des femmes dans l'hérésie: le jugement de saint Jérôme, 241-267; R. Metz, La consécration des vierges dans léglise romaine; L. Mirri, La vita ascetica femminile in san Girolamo; eadem, Il matrimonio in san Girolamo, 365-378; eadem, La dolcezza nella lotta. Donne e ascesi secondo Girolamo; eadem, La verginità nel mistero di Maria in San Girolamo, 325-344; eadem, La teologia dello status monasticus in S. Girolamo, 267-289; eadem, La preghiera delle eremite del deserto, 591-624; eadem, Rapporto tra continenza e matrimonio in S. Girolamo, 303-322; eadem, Girolamo e l'ascesi femminile: dialogo spirituale e santità, 13-23; M. Sanchez, M. del Mar, La visión de la mujer en San Jerónimo a través de su correspondencia, 315-321.

51 A proposito di Paolina, cf. Hieronymus, Epistula 77, 10, CSEL [= Corpus Scriptorum Ecclesiasticorum Latinorum] 55, 47; ibidem, 66, 1-3, CSEL 54, 648-650. Cf. anche L. Mirri, La dolcezza nella lotta, 97-98.

52 Cf. Hieronymus, Epistula 66, 4, CSEL 54, 651-652.

53 Non conosciamo bene la data della nascita di santa Fabiola. Ella morì a Roma il 27 dicembre 399. Fu aristocratica e cristiana. Apparteneva al gruppo delle aristocratiche cristiane di Roma di cui si occupava spiritualmente san Girolamo; esse, sotto il suo influsso, decisero di condurre una vita ascetico-monastica caratterizzata dalle opere di carità verso i poveri e i bisognosi (cf. L. Mirri, La vita ascetica femminile in san Girolamo, passim; eadem, La dolcezza nella lotta, passim). Nel 400, morta Fabiola, Girolamo descrisse la sua vita nell'Epistula 77 indirizzata ad un suo cugino, Oceano. Fabiola apparteneva al famoso casato dei patrizi romani: Gens Fabia. In età molto giovane sposò un uomo dal quale, in seguito, divorziò. Ciò era conforme alle leggi romane, ma urtava contro la legge ecclesiastica. Quando il suo primo marito morì, si risposò. Quando però durante la vigilia pasquale dell'anno successivo anche questo suo sposo morì, Fabiola fece una penitenza pubblica: vestita di sacco entrò nelle Basilica Lateranense, ove fu accolta da papa Siricio che la riconciliò con la Chiesa. Da quel tempo, Fabiola si dedicò all'assistenza dei poveri, dei malati, dei pellegrini 
tus $)^{54}$, alla foce del Tevere ${ }^{55}$. Questa casa accoglieva gratuitamente pellegrini, ammalati e poveri. Si trattava di un xenodochium (dal-

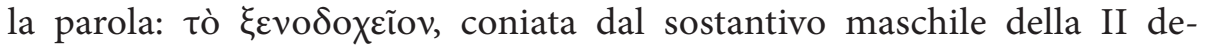
clinazione: ó $\xi \dot{\varepsilon} v o \varsigma=$ "straniero", "ospite" e dal sostantivo neutro

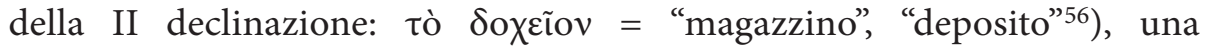

e dei bisognosi, fondando per loro il già menzionato ospedale-rifugio, il quale fu, a Roma, tale istituzione di questo genere in assoluto. la stessa santa Fabiola li serviva. Nel 395, andò a Betlemme per incontrarsi con il suo direttore spirituale, san Girolamo. Entrò nel monastero diretto da santa Paola e condusse la vita monastica approfondendo la Sacra Scrittura. Dopo il conflitto, riguardante Origene, tra Girolamo e Giovanni, vescovo di Gerusalemme ( $\uparrow 417$; cf. Hieronymus, Contra Iohannem Hierosolymitanum), santa Fabiola tornò a Roma. La sua partenza dalla Terra Santa fu anche causata dalla minaccia dei barbari Unni. Fabiola rimase, però, in contatto epistolare con il suo maestro, Girolamo. A proposito di santa Fabiola, cf. Hieronymus, Epistula 77, 1-12, CSEL 55, 38-49. Cf. anche L. Mirri, La dolcezza nella lotta, 125-128.

54 Porto (Portus) era il porto della Roma antica e città situata sulla sponda destra del Tevere, a Nord di Ostia (Ostia Tiberina). Questo porto si estendeva lungo il litorale del Mar Tirreno. Attualmente, esso si trova sul territorio del comune di Fiumicino. Ai tempi di san Pammachio e di santa Fabiola, la città di Porto ottenne lo status di municipio (municipium). Ciò avvenne durante il regno dell'imperatore Costantino il Grande. La città ottenne allora il nome di Civitas Flavia Constantiniana Portuensis, prima si chiamava Portus Ostiae o Portus Augusti. Ai tempi di Costantino, la città fu circondata di mura. Purtroppo, la parte più antica del porto, costruita dall'imperatore Claudio, fu nel IV secolo coperta di sabbia, e nel 408 il porto fu distrutto da Alarico I. La stessa sorte accadde nel 455 durante l'invasione dei Vandali guidati da Genzerico. Ciò nonostante, il porto fu ricostituito dalle rovine; ne parla Cassiodoro. All'inizio del secolo VI, Procopio di Cesarea descrive il porto come una città fiorente. La sua prosperità, però, non durò a lungo, perché nel 537, durante l'invasione degli Ostrogoti guidati da Vitige, fu di nuovo distrutto. A proposito dell'antico porto di Roma, cf.: I. Baldassarre - I. Bragantini - C. Morselli, Necropoli di Porto. Isola Sacra, Roma 1996; S. Nesi, Il Comandante Nesi: un «Alcione» dalle ali spezzate, Milano 2004; S. Keay, M. Millett, L. Paroli, K. Strutt (ed.), Portus. An archaeological survey of the Port of Imperial Rome; L. Di Domenicantonio, Portus; G. Earl, K. Martínez, T. Malzbender, Archaeological Applications of Polynomial Texture Mapping: Analysis, Conservation and Representation, 1-11; S. Keay, L. Paroli (ed.), Portus and its Hinterland: Recent Archaeological Research; S. Keay, G. Boetto (ed.), Portus, Ostia and the Ports of the Roman Mediterranean; S. Keay, Portus and the Alexandrian Grain Trade Revisited; S. Keay (ed.), Rome, Portus and the Mediterranean; S. Keay, G. Earl, F. Felici, P. Copeland, R. Cascino, S. Kay, C. Triantafillou, Interim report on an enigmatic new Trajanic building at Portus, 486-512; S. Keay, R. Cascino, Integrated approaches to the archaeology of Portus, 131-148.

55 Cf. Hieronymus, Epistula 77, 6. 10, CSEL 55, 42-44. 47. Cf. anche L. Mirri, La dolcezza nella lotta, 127.

56 Cf. H.G. Liddell, R. Scott, A Greek-English Lexicon. 
specie di casa del pellegrino (gratuita) unita ad un rifugio ed ospedaletto per poveri ${ }^{57}$.

Nel 398, san Pammachio fece costruire al Celio (a Roma) la basilica in onore dei Santi Giovanni e Paolo (inizialmente la basilica si chiamava titulus Byzantii o Pammachii) ${ }^{58}$. Questa basilica fu costruita sul terreno ove si trovava la casa appartenente alla famiglia di Pammachio, sito in cui, già a partire dal I secolo, si trovava la domus ecclesiae (ciò si vede fino ad oggi nelle parti sotterranee della chiesa $)^{59}$.

\section{Il pasto per i poveri preparato da san Pammachio}

Passiamo ora alla parte principale dellarticolo che riguarda la lettera di san Paolino di Nola indirizzata a san Pammachio. In questa lettera il Vescovo di Nola loda il Senatore-Monaco per la sua carità e misericordia verso i poveri e i bisognosi, per i quali organizzò un ottimo pasto nella Basilica Costantiniana di S. Pietro al Vaticano. La descrizione di questa basilica, oggi quasi del tutto inesistente, ci fornisce preziose informazioni concernenti il suo aspetto.

Qui sotto riportiamo il testo della lettera che ci interessa in modo particolare.

11. [...] tu radunasti nella basilica dell'Apostolo una moltitudine di poveri, i patroni delle nostre anime, che per tutta la città di Roma chiedono l'elemosina per vivere. Ed io, in verità, mi sazio a contemplare lo stupendo spettacolo offerto da questa tua

57 A proposito delle leggi antiche riguardanti lo xenodochium, cf. Iustinianus, Codex 1. 2. 15. 1; idem, Novellae 120. 1.

58 Cf. A. Pollastri, Pammachio, 3789. La basilica del titulus Byzantii o Pammachii viene, per la prima volta, menzionata dagli atti del sinodo romano del 499.

59 A proposito di questa basilica, cf.: M. Armellini, Le Chiese di Roma, dalle loro origini sino al secolo XVI, 276-281; G. Di San Stanislao, La Casa Celimontana dei SS. Martiri Giovanni e Paolo; S. Dell'Addolorata, La Basilica Celimontana dei Santi Giovanni e Paolo; Istituto di Studi Romani, SS. Giovanni e Paolo al Celio; A. Prandi, G Ferrari, The Basilica of Saints John and Paul on the Caelian Hill; G.A. De Sanctis, I Santi Giovanni e Paolo, martiri celimontani; W. Buchowiecki, Handbuch der Kirchen Roms, 125-154; B.M. Margarucci Italiani, Il titolo di Pammachio, Santi Giovanni e Paolo; I. della Portella, Das unterirdische Rom - Katakomben, Bäder, Tempel, 198-205 i 271; L. Zeppegno, R. Mattonelli, Le chiese di Roma, 71-73; A. Englen, Case romane e Antiquarium; H. Brandenburg, Die frühchristlichen Kirchen in Rom vom 4. bis zum 7. Jahrhundert, 164 nn. nonché 323-324; A. Englen, M.G. Filetici, P. Palazzo, C. Pavolini, R. Santolini (ed.), Caelius II. Pars Inferior. Le Case Romane sotto la Basilica dei Ss. Giovanni e Paolo; H.G. Wehrens, Rom - Die christlichen Sakralbauten vom 4. bis zum 9. Jahrhundert - Ein Vademecum, 189-194. 
sì grande opera di carità: mi sembra infatti di vedere tutte queste devote moltitudini di gente misera, gli allievi dell'amore paterno di Dio, affluire a sciami in grandi schiere, fino in fondo all'immensa basilica del glorioso Pietro, attraverso quella veneranda porta regale, il cui frontone da lontano splende con l'azzurra facciata, sicché tutti gli spazi sono gremiti sia all'interno della basilica, sia davanti alle porte dell'atrio, sia davanti ai gradini del sagrato. Vedo i convenuti che si dispongono in ordine, distribuendosi tra le diverse mense e tutti si saziano di cibi abbondanti [...]. 13. Questa gioia arrecavi allo stesso Apostolo, allorquando riempivi tutta la sua basilica di molte schiere di poveri, sia là dove la basilica sotto l'alto tetto si estende vasta lungo la volta centrale, e, risplendendo da lontano col sepolcro dell'Apostolo, abbaglia lo sguardo di coloro che entrano e ne rallegra il cuore, sia là, dove sotto il medesimo imponente tetto essa si estende lateralmente con due ordini di colonne dall'una e dall'altra parte; sia là dove l'atrio splendente si fonde con il vestibolo davanti all'ingresso, nel quale una costruzione a cupola, coperta in alto con tetto di bronzo massiccio, adorna e tiene in ombra la pila di una fontana d'acqua zampillante che serve per lavarci le mani e i volti ${ }^{60}$ con quattro colonne che circondano i getti d'acqua, non senza mistica bellezza. E s'addice invero un siffatto ornamento all'ingresso di una chiesa, di modo che il mistero di salvezza che si compie all'interno sia simboleggiato davanti all'ingresso con una struttura visibile. [...].

15. O Roma, potresti non temere le minacce scagliate contro di te nell'Apocalisse (cf. Ap 17, 1-19, 10), se i tuoi senatori offrissero sempre doni di questo genere. Avresti realmente come vera nobiltà quella che i nostri santi padri Abramo, Isacco e Giacobbe, accoglierebbero nel loro seno paterno [...].

16. Beato te, che non sei intervenuto nel consesso di uomi siffatti e ottieni la lode non sulla cattedra degli empi (cf. Sal 1, 1), bensì nella sede dell'Apostolo e nell'assemblea della Chiesa, cioè nel teatro di Cristo, dove gli spettatori delle gradinate non sono sediziosi, ma benedicenti, e dove Dio stesso è spettatore. Tu dài spettacoli per la Chiesa, non per l'arena dell'anfiteatro, aspirando alla lode eterna, non alla gloria vana. Tu non compri gladiatori né belve, ma ti procuri i mezzi con cui annientare i veri gladiatori, cioè i principi di queste tenebre e i mezzi con cui vincere le vere belve, cioè tutto il potere del diavolo, e schiacciare impunemente sotto i piedi il Leone ed il dragone (cf. Sal $91[90], 13)[\ldots]^{61}$.

60 Al centro dell'atrio, fu collocato un bacino pieno d'acqua (cantharus) o una fontana; prima di entrare nella basilica, i fedeli lì si lavavano le mani e i volti.

61 Paulinus Nolanus, Epistula 13, 11. 13. 15-16, CSEL 29, 92-97 (tr. italiana: G. Santaniello [ed.], Paolino di Nola. Le Lettere [= "Strenae Nolanae. Collana di studi etici"], Marigliano [NA] 1992, 419-423: "11. Itaque patronos animarum nostrarum pauperes, qui tota Roma stipem meritant, multitudinem in aula apostoli congregasti. Pulchro equidem tanti operis tui spectaculo pascor. Videre enim mihi videor tota illa religiosa miserandae plebis examina, illos pietatis divinae alumnos tantis influere penitus agminibus in amplissimam gloriosi Petri basilicam per illam venerabilem regiam cerula eminus fronte ridentem, ut tota et intra basilicam et pro ianuis atrii et pro gradibus campi spatia coartentur. Video congregatos ita distincte per accubitus ordinari et profluis omnes saturari cibis [...]. 
Come si vede dal testo citato, Pammachio preparò il ricevimento per i poveri di Roma non altrove, ma addirittura nel luogo più importante per i cristiani come tali, e per i Cristiani della Città Eterna in particolare: "Nel tempio [letteralmente: nell'aula, nella sala] dell'Apostolo"62, ovverosia: nella Basilica di S. Pietro in Vaticano, costruita dall'imperatore Costantino il Grande sopra la tomba del Principe degli Apostoli.

Infatti, la politica dell'imperatore Costantino il Grande e di Licinio, iniziata con l'“Editto di Milano" nel 313, diede ai Cristiani la libertà del loro culto e proclamò la loro religione religio licita, ossia: riconosciuta dalle leggi dell'Impero Romano. Durante il primo concilio ecumenico di Nicea nel 325, Costantino si

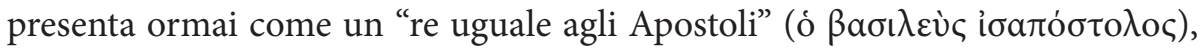
anche se in quel periodo egli era ancora soltanto un catecumeno (nota bene: sarà battezzato moribondo, a Nicomedia, nel 337, e il battesimo gli sarà conferito dal vescovo di quella città, Eusebio, noto capo degli eretici ariani).

Essendo catecumeno, Costantino cominciò a costruire delle imponenti chiese cristiane, site nei posti più cari ai fedeli di Cristo: in Palestina e a Roma. La più antica basilica da lui costruita è quella di S. Giovanni in Laterano, a Roma. Comunque, la più nota e la più splendida di esse fu, senza dubbio, quella di S. Pietro in Vaticano: ebbe 119 metri di lunghezza, 63 di larghezza e 37 metri di altezza. Essa fu costruita sul luogo della sepoltura di san Pietro Apostolo. Precedentemente (dal II al IV secolo), sulla tomba del Principe degli Apostoli si ergeva una piccola chiesetta (memoria), edificata dai primissimi

13. Quanto ipsum apostolum adtollebas gaudio, cum totam eius basilicam densis inopum coetibus stipavisses, vel qua sub alto sui culminis mediis ampla laquearibus longum patet et apostolico eminus solio coruscans ingredientium lumina stringit et corda laetificat, vel qua sub eadem mole tectorum geminis utrimque porticibus latera diffundit, quave praetento, nitens atrium, fusa vestibulo est, ubi cantharum ministra manibus et oribus nostris fluenta ructantem fastigatus solido aere tholus ornat et inumbrat, non sine mystica specie quattuor columnis salientes aquas ambiens. Decet enim ingressum ecclesiae talis ornatus, ut quod intus mysterio salutari geritur spectabili pro foribus opere signetur. [...]. 15. Poteras, Roma, illas intentas in apocalypsi minas non timere, si talia semper ederent munera senatores tui. Vere tunc tibi nobilis esset illa nobilitas, quam sacrati patres Abraham Isaac et Iacob paternis sinibus exciperent [...]. 16. Beatus, qui non adisti in tale consilium nec in cathedra pestilentiae, sed in apostoli sede et in ecclesiae coetu id est Christi theatro non seditiosis sed benedicentibus cuneis deo ipso spectatore laudaris, ecclesiae munerarius, non harenae nec inanis gloriae sed aeternae laudis ambitor. Non gladiatores nec belvas emis, sed quibus veros bestias hoc est omnem zabuli virtutem superes et presso inpune vestigio conculces leonem et draconem".

62 Paulinus Nolanus, Epistula 13, 11, CSEL 29, 92: “[...] in aula apostoli congregasti”. 
Cristiani ai margini del circo di Gaio e di Nerone (circus Gai et Neronis), ai piedi del colle Vaticano ${ }^{63}$.

Non conosciamo esattamente la cronologia della costruzione della prima basilica vaticana. Il Liber Pontificalis informa che fu costruita da Costantino I (il Grande) durante il pontificato di papa Silvestro I (314-335) ${ }^{64}$. I lavori per la sua costruzione furono probabilmente iniziati tra il 319 e il 326 ed ultimati circa nel 333. Non è, però, escluso che qualche lavoro di finitura si sia potuto estendere dopo la data della morte di san Silvestro (il 335), o persino dello stesso imperatore Costantino il Grande (il 337) ${ }^{65}$.

Per costruire la basilica, Costantino, sostenuto probabilmente da papa Silvestro, sotterrò quasi tutti i mausolei pagani e le tombe del cimitero che, fino a tale operazione, era ancora del tutto funzionante, infrangendo in tal modo le vigenti leggi e le consuetudini e tradizioni sia laiche che religiose ${ }^{66}$. Tale comportamento dell'Imperatore può essere giustificato soltanto dalla certezza dell'autenticità della tomba del Principe degli Apostoli ${ }^{67}$. Le informazioni in proposito ci sono state fornite, per la prima volta, da Eusebio di Cesarea, sulla base di unantica tradizione e facendo appello alla testimonianza di un chierico di nome Gaio ${ }^{68}$ :

[...] al tempo di Nerone, proprio a Roma Paolo venne decapitato e Pietro crocifisso. Il nome di Pietro e Paolo, giunto fino ai nostri giorni sulle loro tombe, che si trovano a Roma, attesta la veridicità di questa storia, e così pure un uomo ecclesiastico, di nome Gaio, che visse al tempo di Zefirino, vescovo di Roma. Egli, disputando

63 Cf. M. Ghilardi, Vaticano, in A. Di Berardino (ed.), Nuovo dizionario patristico e di antichità cristiane, III, 5550.

64 Cf. Liber Pontificalis (Pars prior) 34, 16, in Th. Mommsen (ed.), Gestorum Pontificum Romanorum Liber Pontificalis, 56-57: "Eodem tempore Augustus Constantinus fecit ex rogatu Silvestri episcopi basilicam beato Petro apostolo [...]".

65 Cf. L. Zeppegno, R. Mattonelli, Le chiese di Roma, 12-14.

66 Cf. A. Angela, San Pietro. Segreti e meraviglie in un racconto lungo duemila anni, passim.

67 Cf. La Fabbrica di San Pietro in Vaticano, Su questa pietra, catalogo della mostra, passim.

68 Gaio era un teologo romano che ai tempi di papa san Zefirino (198-217) scrisse un'opera contro i montanisti intitolata "Dialogo con il montanista Proclo". Di questopera, sono giunti a noi soltanto pochi frammenti citati nella "Storia ecclesiastica" di Eusebio di Cesarea (cf. Eusebius Caesariensis, Historia ecclesiastica III 28, 1-2; III 31, 4; VI 20, 3). Un'informazione riguardante Gaio ci viene anche fornita da Fozio ( $\Phi \dot{\omega} \tau \iota$; ;f. Photius, Bibliotheca 98). 
nei suoi scritti con Proclo, capo della setta dei Frigi ${ }^{69}$, dice queste cose sui luoghi che custodiscono le sacre spoglie dei suddetti apostoli: "Io sono in grado di mostrare i trofei degli apostoli; andando infatti al Vaticano o lungo la via Ostiense, vi troverai i trofei di quelli che hanno fondato questa Chiesa"70.

Il terreno, quindi, sul quale Costantino fece costruire la basilica e sul quale oggi è situata l'attuale basilica vaticana, fu all'inizio considerato il luogo della sepoltura di san Pietro Apostolo, crocifisso proprio nelle vicinanze dei giardini di Nerone (Horti Neroniani) ${ }^{71}$.

La basilica costantiniana come tale era circondata, dalla parte della facciata, da un grande nartece (nartex), cioè un portico a quattro lati e a cielo aperto, documentato nel $397 \mathrm{e}$, perciò, costruito probabilmente già qualche tempo dopo la fine della costruzione della basilica come tale. Ad esso conduceva una grande ed ampia scalinata ${ }^{72}$. Senza dubbio, a questo luogo pensava san Paolino scrivendo:

[...] mi sembra di vedere tutte queste devote moltitudini di gente misera, gli allievi dell'amore paterno di Dio, affluire a sciami in grandi schiere, fino in fondo all'immensa basilica del glorioso Pietro, attraverso quella veneranda porta regale, il cui frontone da lontano splende con l'azzurra facciata, sicché tutti gli spazi sono gremiti sia all'interno della basilica, sia davanti alle porte dellatrio, sia davanti ai gradini del sagrato"73.

I numerosi poveri di Roma entravano alla basilica attraverso la porta principale il cui frontone, come leggiamo nel testo citato, aveva colore azzurro. La basilica vera e propria, invece, era preceduta da un atrio al quale si accedeva attraverso porte alle quali menava una scalinata. San Paolino collocò i commensali attorno a molti tavoli e tutta la basilica del Principe degli Apostoli si riempì di quella "fitta folla dei poveri". Molti di essi stavano seduti "sotto l'alto

69 Essi erano montanisti. Venivano anche chiamati "catafigi", e ciò dal luogo dell'inizio della loro dottrina eterodossa.

70 Eusebius Caesariensis, Historia ecclesiastica II 25, 5-7 (tr. italiana: Eusebio di Cesarea, Storia ecclesiastica/1, 132-133).

71 Cf. L. Bianchi, Ad limina Petri: spazio e memoria della Roma cristiana, passim; M. Ghilardi, Vaticano, 5550.

72 Cf. P. Testini, Le catacombe e gli antichi cimiteri Cristiani in Roma, 101-105.

73 Paulinus Nolanus, Epistula 13, 11, CSEL 29, 93: "Videre enim mihi videor tota illa religiosa miserandae plebis examina, illos pietatis divinae alumnos tantis influere penitus agminibus in amplissimam gloriosi Petri basilicam per illam venerabilem regiam cerula eminus fronte ridentem, ut tota et intra basilicam et pro ianuis atrii et pro gradibus campi spatia coartentur". 
tetto" della basilica "sotto la volta centrale", ossia vicino alla tomba dell'Apostolo Pietro. Gli altri poveri, invece, erano collocati lì ove la basilica "sotto il medesimo imponente tetto si estende lateralmente con due ordini di colonne dall'una e dall'altra parte; sia là dove l'atrio splendente si fonde con il vestibolo davanti all'ingresso". Questo atrio, invece, "si fonde con il vestibolo davanti all'ingresso, nel quale una costruzione a cupola, coperta in alto con tetto di bronzo massiccio, adorna e tiene in ombra la pila di una fontana d'acqua zampillante che serve per lavarci le mani e i volti con quattro colonne che circondano i getti d'acqua, non senza mistica bellezza".

Lantica basilica vaticana era lunga di 120 metri ed aveva cinque navate, divise l'una dall'altra dalle alte colonne con i capitelli corinzi. Il frontone della basilica, avente tre porte, conduceva ad un atrio formato da quattro portici. Latrio era così bello che veniva chiamato "Paradiso" (Paradisus). Al centro di esso, come leggiamo nel testo citato, era collocata una fontana di bronzo a forma di pigna alta ben quattro metri. Sopra la fontana si stendeva un baldacchino appoggiato su otto colonne, ornate con bei pavoni di bronzo dorato e, probabilmente, anche con delle sculture a forma di delfini.

La basilica non veniva mai chiusa, ma era accessibile ventiquattro ore su ventiquattro. Le celebrazioni papali, all'epoca, erano molto più rare rispetto ai nostri tempi. In essa, perciò, venivano anche celebrati riti di provenienza pagana, ai quali i Cristiani diedero dei contenuti e dei significati di stampo evangelico. Il più frequente di essi era un cosiddetto refrigerium, ovverosia un pasto funebre celebrato nell'anniversario della morte di un defunto. A volte, a tale refrigerium partecipavano persone così numerose che riempivano non solamente la basilica, ma anche l'atrio, la scalinata e la piazza sottostante. Per tale ragione, il pasto preparato nella basilica vaticana ai poveri da san Paolino non veniva scorto e giudicato dai cristiani come un comportamento sconveniente o strano, benché questo pasto, come pare, non avesse niente in comune con un refrigerium.

Descrivendo l'accesso alla basilica e al suo atrio, san Paolino afferma: "s'addice invero un siffatto ornamento all'ingresso di una chiesa, di modo che il mistero di salvezza che si compie all'interno sia simboleggiato davanti all'ingresso con una struttura visibile"74.

Continuando a lodare la carità di san Pammachio verso i poveri e i bisognosi, il Vescovo di Nola apostrofa retoricamente la città di Roma:

74 Paulinus Nolanus, Epistula 13, 13, CSEL 29, 95: "Decet enim ingressum ecclesiae talis ornatus, ut quod intus mysterio salutari geritur spectabili pro foribus opere signetur". 
O Roma, potresti non temere le minacce scagliate contro di te nell'Apocalisse (cf. Ap 17, 1 - 19, 10), se i tuoi senatori offrissero sempre doni di questo genere. Avresti realmente come vera nobiltà quella che i nostri santi padri Abramo, Isacco e Giacobbe, accoglierebbero nel loro seno paterno [...]. [...]. Beato te, che non sei intervenuto nel consesso di uomi siffatti e ottieni la lode non sulla cattedra degli empi (cf. Sal 1, 1), bensì nella sede dell'Apostolo e nell'assemblea della Chiesa, cioè nel teatro di Cristo, dove gli spettatori delle gradinate non sono sediziosi, ma benedicenti, e dove Dio stesso è spettatore. Tu dài spettacoli per la Chiesa, non per l'arena dell'anfiteatro, aspirando alla lode eterna, non alla gloria vana. Tu non compri gladiatori né belve, ma ti procuri i mezzi con cui annientare i veri gladiatori, cioè i principi di queste tenebre e i mezzi con cui vincere le vere belve, cioè tutto il potere del diavolo, e schiacciare impunemente sotto i piedi il Leone ed il dragone (cf. Sal $91[90], 13)^{75}$.

Come sappiamo, Ap 17 presenta e rimprovera l'impero di peccato e di malvagità. La grande meretrice, ivi descritta, porta impronte della Roma pagana, peccatrice con la sua dissolutezza, malvagità, ingiusta ricchezza, spudoratezza e con il culto divino usurpato dagli imperatori pagani. Tutto ciò si oppone a Dio e alle sue leggi. Un'alleata di Roma nella vita peccaminosa e dissoluta contraria a Dio è anche la meretrice Babilonia, rea del sangue innocente dei testimoni di Gesù. Queste forze nemiche di Dio combatteranno contro il Divino Agnello, ma saranno da lui vinte. Insieme all'Agnello, Re dei re e Signore eterno, trionferanno coloro che credono in lui e gli appartengono. La meretrice, invece, e i suoi alleati saranno distrutti. Anche la Bestia e i suoi alleati combatteranno contro la stessa meretrice. Infatti, satana distrugge anche i propri alleati, dei quali si serviva per attuare i suoi perfidi scopi. Dio, però, soltanto per un certo tempo permette gli intrighi e gli attacchi di satana ${ }^{76}$.

Appellandosi a questo testo dell'Apocalisse, san Paolino critica e stigmatizza i crudeli spettacoli pagani organizzati nei teatri e nei circhi romani. Bisogna aggiungere che la posizione dei Padri della Chiesa rispetto agli spettacoli pagani, e specialmente quelli teatrali, era molto negativa, dato che essi presentavano

75 Paulinus Nolanus, Epistula 13, 15-16, CSEL 29, 96-97: "Poteras, Roma, illas intentas in apocalypsi minas non timere, si talia semper ederent munera senatores tui. Vere tunc tibi nobilis esset illa nobilitas, quam sacrati patres Abraham Isaac et Iacob paternis sinibus exciperent [...]. Beatus, qui non adisti in tale consilium nec in cathedra pestilentiae, sed in apostoli sede et in ecclesiae coetu id est Christi theatro non seditiosis sed benedicentibus cuneis deo ipso spectatore laudaris, ecclesiae munerarius, non harenae nec inanis gloriae sed aeternae laudis ambitor. Non gladiatores nec belvas emis, sed quibus veros bestias hoc est omnem zabuli virtutem superes et presso inpune vestigio conculces leonem et draconem".

76 Cf. Pismo Święte Starego i Nowego Testamentu, 2889. 
scene immorali, pornografiche e, di regola, si basavano sulla mitologia pagana. Tali spettacoli deridevano spesso i Cristiani e lo stesso Gesù Cristo ${ }^{77}$.

A questi cruenti e dissoluti spettacoli, san Paolino oppone gli "spettacoli per la Chiesa", organizzati da Pammachio nel "teatro di Cristo", cioè nella Basilica di S. Pietro. Si trattava di un "teatro" completamente diverso da quello pagano. Nel "teatro di Cristo"78, infatti, "gli spettatori delle gradinate non sono sediziosi, ma benedicenti"79. Lì anche "Dio stesso è spettatore" 80 di questi peculiari spettacoli atti a servire e ad aiutare il prossimo, o più precisamente: a servire l'altro, ad aiutare l'uomo bisognoso, vedendo in lui lo stesso Gesù Cristo. Proprio tale dimensione ebbe il pasto organizzato da san Pammachio descritto e lodato dal Vescovo di Nola.

\section{Conclusione}

Nell'articolo abbiamo voluto presentare un piccolo gioiello dellepistolografia patristica: un frammento della Lettera XIII di san Paolino di Nola indirizzata a san Pammachio. Questo testo descrive il pasto che san Pammachio preparò per i poveri di Roma nel tempo in cui era senatore. La data precisa dell'episodio è a noi sconosciuta. Sappiamo soltanto che ciò avvenne nelletà piuttosto avanzata di Pammachio, quando sua moglie Terasia era ormai defunta ed egli stesso, desideroso di vivere da bravo monaco in povertà, distribuiva i suoi beni ai bisognosi.

Paolino dovette essere molto impressionato dal pasto predisposto da Pammachio, dato che lo descrisse abbastanza dettagliatamente e ringraziò il Monaco-Senatore per esso.

Per introdurre alla descrizione del pasto e per mostrare tutto il suo contesto, prima di analizzare il brano preso in esame, abbiamo presentato brevemente ambo i Santi: dapprima l'Autore della lettera e poi anche il suo Destinatario. Come possiamo constatare, i due Santi avevano in comune tante caratteristi-

77 A proposito di questo tema, cf.: O. Pasquato, Spettacoli, 3279-3284; S. Longosz, święty Augustyn a starożytny dramat teatralny, 369-394, 859-879; idem, Czy Paweł Apostoł cytowat komediopisarza Menandra?, 907-924; idem, Atellana w okresie patrystycznym, 273-291. Per quanto riguarda un'antologia di testi patristici contro gli spettacoli teatrali, cf. S. Longosz, Widowiska teatralne zagrożeniem dla życia rodzinnego według św. Jana Chryzostoma, 161-198.

78 Paulinus Nolanus, Epistula 13, 16, CSEL 29, 97: “[...] in Christi theatro [...]”.

79 Paulinus Nolanus, Epistula 13, 16, CSEL 29, 97: “[...] non seditiosis sed benedicentibus cuneis $[\ldots] "$.

80 Paulinus Nolanus, Epistula 13, 16, CSEL 29, 97: “[...] deo ipso spectatore [...]”. 
che peculiari e, anche se Paolino era più giovane rispetto a Pammachio di una ventina di anni, essi nutrivano desideri simili e, come pare, mantenevano uno stretto contatto di amicizia. D'altra parte, entrambi furono uniti dalla santità che si concretizzò, nella loro vita monastica, legata al servizio per i poveri e i bisognosi che essi aiutavano con i loro beni famigliari.

Il brano della lettera preso in esame permette di constatare che non si trattò qui di una semplice filantropia in senso laico della parola, ma di un'idea molto più profonda, basata sul Vangelo e sull'osservazione del crudele mondo pagano di allora atto, di regola, a soddisfare desideri egoistici ed edonistici dellessere umano privo di altruismo e di alti ideali (anche pagani!). Proprio in tale contesto san Paolino vede il pasto preparato da Pammachio come una vera "gara per la Chiesa" e lo oppone agli spettacoli pagani. Anche la Basilica di S. Pietro, nella quale si svolse il pasto, chiama conseguentemente con l'espressione di "teatro di Cristo", rafforzando anche di più, in tal modo, la stessa contrapposizione.

Il brano analizzato possiede anche un notevole valore per gli storici e gli archeologi, perché fornisce numerosi particolari concernenti l'aspetto della primissima Basilica di S. Pietro in Vaticano costruita dall'imperatore Costantino il Grande. La descrizione, contenuta nella lettera, conferma l'impotenza e la grandezza di quest'edificio sacro così importante per tutta la cristianità che perdurerà sino all'inizio del secolo XVI, quando papa Giulio II deciderà di costruire sullo stesso luogo una nuova, ancora più imponente basilica dedicata al Principe degli Apostoli.

\section{Bibliografia}

Akar Ph., Camille et la Concorde, Hypothèses 5 (2002), 205-215.

Albert G., Goten in Konstantinopel: Untersuchungen zur oströmischen Geschichte um das Jahr 400 n.Chr., Paderborn 1984.

Alexanderson B., Quelques idées sur le texte et l'interprétation des cuvres de Julien d'Éclane Expositio libri Iob et Tractatus prophetarum Osee, Iohel et Amos, avec des remarques sur le texte et l'interprétation des commentaires de Jérôme sur les Douze Prophètes, Göteborg 2011.

Alföldi A., Der Vater des Vaterlandes im römischen Denken [= Reihe Libelli, 261], Darmstadt 1971.

Amann É., Nicétas de Rémésiana, in A. Vacant, E. Mangenot, É. Amann (ed.), Dictionnaire de Théologie Catholique, XI/1, Paris 1930, 477-479.

Ambrosius Mediolanensis, Epistula.

Angela A., San Pietro. Segreti e meraviglie in un racconto lungo duemila anni, Milano 2015. 
Antin P., Le monachisme selon saint Jérôme, in Mélanges bénédictins publiés à loccasion du XIVecentenaire de la morte de S. Benoît par les moines de l'Abbaye de S. Jérôme de Rome, Abbaye de S. Wandrille 1947, 71-118.

Arjava A., Jerome and women, Arctos. Acta philologica Fennica 23 (1989), 5-18.

Armellini M., Le Chiese di Roma, dalle loro origini sino al secolo XVI, Roma 1887, 276-281 .

Aste A., (ed.), Gli epigrammi di papa Damaso I [= Collana Università \& Ricerca], Tricase (LE) 2014.

Augustinus Hipponensis, Epistulae, CSEL 34/1-2.

Aurelius Augustinus, De Civitate Dei.

Ausbüttel F.M., Germanische Herrscher, Darmstadt 2007.

Balboni D., Fabiola, in Bibliotheca Sanctorum, V, Roma 1983.

Baldassarre I., Bragantini I., Morselli C., Necropoli di Porto. Isola Sacra, Roma 1996.

Balsdon J.P.V.D., Pater patriae, in N.G. Lemprière Hammond - H.H. Scullard (ed.), 1590.

Barclift L., In Controversy with Saint Augustine: Julian of Aeclanum on the Nature of Sin, Recherches de théologie ancienne et médiévale 58 (1991), 5-20.

Bardenhewer O., Geschichte der altkirchlichen Literatur, III, Freiburg im Breisgau 1923.

Bautz F.W., Ausonius, Decimus Magnus, in Biographisch-Bibliographisches Kirchenlexikon, II, Hamm 1990, 304-305.

Bautz F.W., Gratian, in Biographisch-Bibliographisches Kirchenlexikon, II, Hamm 1990, 289-290.

Bayet J., Appendice à lédition de Tite Live, Histoire romaine, V, Paris 1969.

Bianchi L., Ad limina Petri: spazio e memoria della Roma cristiana [= Saggi. Arti e lettere], Roma 1999.

Blasen Ph., Nicetas of Remesiana - A Missionary Bishop in Dacia?, Studia Universitatis Babeș-Bolyai Theologia catholica, 1-2 (2012), 39-49.

Bodart E., Rufinus von Aquileia, in Lexikon des Mittelalters, VII, München 1995, 1088-1089 .

Booth A.D., Quelques dates hagiographiques: Mélanie l’Ancienne, Saint Martin, Mélanie la Jeune, Phoenix 37/2 (1983), 144-151.

Bowman G., Des Julian von Aeclanum Kommentar zu den Propheten Osee, Joel und Amos. Ein Beitrag zur Geschichte der Exegese [= Analecta Biblica 9], Roma 1958.

Brandenburg H., Die frühchristlichen Kirchen in Rom vom 4. bis zum 7. Jahrhundert, Regensburg 2013.

Brennecke H.C., Nicetas von Remesiana, in Theologische Realenzyklopädie, XXIV, Berlin-New York 1994, 460-463.

Breukelaar A., Martinus von Tours, in Biographisch-Bibliographisches Kirchenlexikon, V, Herzberg 1993, 949-955.

Bruckner A., Julian von Eclanum. Sein Leben und seine Lehre [= Texte und Untersuchungen zur Geschichte der altchristlichen Literatur, 15], Leipzig 1897.

Brunot A., Sainte Paule et Sainte Eustochium, Jérusalem 30 (1964), 259-264.

Buchowiecki W., Handbuch der Kirchen Roms. Der römische Sakralbau in Geschichte und Kunst von der altchristlichen Zeit bis zur Gegenwart, II, Wien 1970, 125-154. 
Burck E., Die Erzählungskunst des T. Livius, Berlin 1934.

Burke E., St. Jerome as a Spiritual Director, in A Monument to St. Jerome, New York 1952, 145-169.

Burn A.E., Niceta of Remesiana. His Life and Works, Cambridge 1905.

Caraffa F., Eustochio, in Bibliotheca Sanctorum, V, Roma 1983, 302-304.

Carletti C., Damaso I, in M. Bray (ed.), Enciclopedia dei Papi, I, Roma 2000, 349-372.

Caroline P., Bammel H., Der Römerbrieftext des Rufin und seine Origenes-Übersetzung, Freiburg im Breisgau 1985.

Cecchetti H., Hieronymus ab Aventino in Bethlehemiticum coenobium da un libro di prossima pubblicazione: San Girolamo e lo studio delle Divine Scritture, Latinitas 11 (1963), 4.

Chisholm H., Rufinus, Tyrannius, in Encyclopaedia Britannica, XXIII, Cambridge $1911^{11}, 820-821$.

Cipriani N., Aspetti letterari dell'Ad Florum di Giuliano d'Eclano, Augustinianum 15 (1975), 125-167.

Cipriani N., Echi antiapollinaristici e aristotelismo nella polemica di Giuliano d'Eclano, Augustinianum 21 (1981), 373-389.

Clavis Patrum Latinorum 646-652) furono pubblicati in Patrologiae Latinae cursus completus 52, 837-876.

Coşkun A., Die gens Ausoniana an der Macht. Untersuchungen zu Decimius Magnus Ausonius und seiner Familie [= Prosopographica et Genealogica, 8], Oxford 2002.

Costanza S., Ricci C., Paolino di Nola, in A. Di Berardino (ed.), Nuovo dizionario patristico e di antichità cristiane, III, Genova-Milano 2008.

Courcelle P., Histoire littéraire des grandes invasions germaniques, Paris 1964², 31-77.

Croke B., Arbogast and the Death of Valentinian II, Historia 25 (1976), 235-244.

Crouzel H., Les échanges littéraires entre Bordeaux et l'Orient au IV siècle, Revue d'histoire du livre 3 (1973), 316-319.

Crouzel H., Odrobina L., Amando di Bordeaux, in A. Di Berardino (ed.), Nuovo dizionario patristico e di antichità cristiane, I, Genova-Milano 2006, 225-226.

de Montcheuil Y., La polémique de saint Augustine contre Julien d'Éclane d'après l'Opus imperfectum, Recherches de science religieuse 44 (1956), 193-218.

de Sainte-Marthe D., Gallia Christiana, II, Parisiis 1720.

De Sanctis G.A., I Santi Giovanni e Paolo, martiri celimontani, Isola del Liri 1962.

Degórski B., Il mistero dell'incarnazione nel commento di san Niceta di Remesiana al «Simbolo Apostolico», Angelicum 87 (2010), 231-237.

Degórski B., Il monachesimo latino nel Mediterraneo (secc. IV-VI): protagonisti e legislazione [= Katolicki Uniwersytet Lubelski Jana Pawła II. Instytut Badań nad Antykiem Chrześcijańskim], Lublin 2006, 117-118.

Degórski B., Katechezy przedchrzcielne Nicetasa z Remezjany, Vox Patrum 10 (1990), 107-111.

Degórski B., Korzenie duchowości paulińskiej. Pierwsi mnisi egipscy, in J.A. Nalaskowski, A. Napiórkowski (ed.), W poszukiwaniu duchowości paulińskiej. Studium historyczno-teologiczne, Kraków 1999, 11-65. 
Degórski B., L'«abate» come pastore in epoca teodosiana, in Vescovi e pastori in epoca teodosiana. In occasione del XVI centenario della consacrazione episcopale di S. Agostino, 396-1996. XXV Incontro di studiosi dell'antichità cristiana. Roma, 8-11 maggio 1996 [= Studia Ephemeridis Augustinianum, 58], I, Roma 1997, 91-120 (in particolare pp.: 98-103; 107-109).

Degórski B., Najstarszy monastycyzm żeński, Dissertationes Paulinorum 12 [2003].

Degórski B., Opaci okresu Teodozjańskiego, Dissertationes Paulinorum 9 (1996), 5-51 (specialmente le pagine: 15-24; 31-33).

Degórski B., św. Hieronim. Listy do Eustochium. Listy 22, 31, 108 [= źródła monastyczne, 33], Tyniec-Kraków 2004 (passim).

Del Ton G., Paola di Roma, in Bibliotheca Sanctorum, X, Roma 1982, 123-136.

Dell'Addolorata S., La Basilica Celimontana dei Santi Giovanni e Paolo, Roma 1930. Istituto di Studi Romani, SS. Giovanni e Paolo al Celio [= Chiese di Roma, cenni religiosi, storici, artistici, 70], Roma 1956.

della Portella I., Das unterirdische Rom - Katakomben, Bäder, Tempel. Könemann, Köln 2000.

Demogeot J., Études sur Ausone, Bordeaux 1838.

Demougeot E., La formation de l'Europe et les invasions barbares, 2, De l'avènement de Dioclétien (284) à l'occupation germanique de l'Empire Romain d'Occident (debut du VI siècle), II, Paris 1979, 451-464.

Di Berardino A., La poesia cristiana, in idem (ed.), Patrologia, III: Dal Concilio di Nicea (325) al Concilio di Calcedonia (451). I Padri latini, Casale Monferrato 1978.

Di Domenicantonio L., Portus, Milano 2008.

Di San Stanislao G., La Casa Celimontana dei SS. Martiri Giovanni e Paolo, Roma 1894. Drączkowski F., Patrologia, Pelplin 1998.

Drobner H.R., Rufinus, Tyrannius, in Biographisch-Bibliographisches Kirchenlexikon, VIII, Herzberg 1994, 959-972.

Drouve A., Der heilige Martin. Patron der Armen - Vorbild der Nächstenliebe, Kevelaer 2011.

Drumm J. (ed.), Martin von Tours. Der Lebensbericht von Sulpicius Severus, Ostfildern 1997.

Dumézil G., Camillus: A Study of Indo-European Religion as Roman History, University of California Press 1980.

Dumézil G., Mythe et épopée, III, Paris 1995, 1163-1271 e 1288-1310.

Duval Y.-M., Julien d'Éclane et Rufin d'Aquilée. Du concile de Rimini à la répression pélagienne. L'intervention imperial en matière religieuse, Revue des études augustiniennes 24 (1978), 243-271.

Earl G., Martínez K., Malzbender T., Archaeological Applications of Polynomial Texture Mapping: Analysis, Conservation and Representation, Journal of Archaeological Science 30 (2010), 1-11.

Eder W., Furius [I 13], in Der Neue Pauly, IV, Stuttgart 1998, 715-716.

Eigler U., Camillus, in P. von Möllendorff, A. Simonis, L. Simonis (ed.), Historische Gestalten der Antike. Rezeption in Literatur, Kunst und Musik [= Der Neue Pauly. Supplemente, 8], Stuttgart-Weimar 2013, 241-246. 
Englen A., Case romane e Antiquarium: sotto la Basilica dei SS. Giovanni e Paolo al Celio: guida breve, Roma 2004.

Englen A., Filetici M.G., Palazzo P., Pavolini C., Santolini R. (ed.), Caelius II. Pars Inferior. Le Case Romane sotto la Basilica dei Ss. Giovanni e Paolo [= Palinsesti Romani, 2]. Roma 2015.

Enßlin W., Valentinianus II, in Paulys Realencyclopädie der classischen Altertumswissenschaft, VII A/2, Stuttgart 1948, 2205-2207.

Étienne R., Bordeaux antique, Bordeaux 1962.

Eusebius Caesariensis, Historia ecclesiastica II 25, 5-7 (tr. italiana: Eusebio di Cesarea, Storia ecclesiastica/1, introduzione a cura di F. Migliore, traduzione e note a cura di S. Borzì [= Collana di testi patristici, 158], Roma 2001, 132-133).

Eusebius Caesariensis, Historia ecclesiastica III 28, 1-2; III 31, 4; VI 20, 3.

Eutropius, Breviarium ab Urbe condita 1, 20.

Fabre P., Essai sur la chronologie de l'ouvre de s. Paulin de Nole, Paris 1948.

Fabre P., Paulin de Nole et l'amitié chrétienne, Paris 1949.

Fabre P., St. Paulin de Nole et l'amitié chrétienne [= Bibliothèque des Écoles Françaises d'Athènes et de Rome, 167], Paris 1949.

Fedalto G., Rufino di Concordia (345 s. 410-411): tra Oriente e Occidente, Roma 1990.

Fedalto G., Rufino di Concordia tra Oriente e Occidente, Roma 2005.

Ferrua A., Epigrammata Damasiana, Città del Vaticano 1942.

Flusin B., Melania die Ältere, in Lexikon des Mittelalters, VI, München-Zürich 1993.

Fontaine J., Martin, hl., in W. Kasper (ed.), Lexikon für Theologie und Kirche, VI, Freiburg im Breisgau 19976 $1427-1428$.

Fontaine J., Vérité et fiction dans la chronologie de la Vita Martini, in Saint Martin et son temps [= Studia Anselmiana, 46], Roma 1961, 189-236.

Fros H., Sowa F., Księga imion i świętych, II: D-G, Kraków 1997.

Gagé J., La Némésis de Camille, Revue des Études Latines 50 (1972), 111-138.

Gamber K., Fragen zu Person und Werk des Bischof Niceta von Remesiana, Römische Quartalschrift 62 (1967), 222-231.

Gamber K., Ist Niceta von Remesiana der Verfasser des pseudoambrosianischen Sermo De Spiritu Sancto?, Ostkirchliche Studien, 11 (1962), 204-206.

Gamber K., Ist Niceta von Remesiana der Verfasser von De Sacramentis?, Ostkirchliche Studien 7 (1958), 153-172.

Gamber K., Niceta von Remesiana. Instructio ad competentes [= Textus Patristici et Liturgici, 1, 2, 5, 7], Regensburg 1964, 1965, 1966, 1969.

Gatto L., Storia di Roma nel Medioevo, Roma 2000.

Ghilardi M., Vaticano, in A. Di Berardino (ed.), Nuovo dizionario patristico e di antichità cristiane, III, Genova - Milano 2008, 5550.

Gobry I., Storia del monachesimo. Le origini orientali: da sant'Antonio a san Basilio. Il radicamento in Occidente: da san Martino a san Benedetto, I, Roma 1991.

Gordini G.D., Forme di vita ascetica a Roma nel IV secolo, Scrinium Theologicum 1 (1953), 7-57.

Gordini G.D., Il monachesimo romano in Palestina nel IV secolo, in Saint Martin et son temps [= Studia Anselmiana, 46], Roma 1961, 85-107. 
Gordini G.D., L'opposizione al monachesimo a Roma nel IV secolo, in Dalla Chiesa antica alla Chiesa moderna [= Miscellanea Historiae Pontificiae], Roma 1983, 19-35.

Gordini G.D., Origine e sviluppo del monachesimo a Roma, Gregorianum 37 (1956), 220-260.

Gordini G.D., Marcella di Roma, in Bibliotheca Sanctorum, VIII, Roma 1967.

Górny J., Udział wspólnot monastycznych $w$ rozwoju życia intelektualnego i ascetycznego Kościoła na przełomie IV-V wieku w świetle pism św. Hieronima, Studia Warmińskie 18 (1981), 537-578.

Gottlieb G., Gratianus, in Reallexikon für Antike und Christentum, XII, Stuttgart 1983, 718-732.

Green M., The Supporters of the Antipope Ursinus, Journal of Theological Studies 22 (1971), 531-538.

Grego I., Betlemme, dolce patria mia!, Bibbia e Oriente 22 (1980), 63-84.

Gribomont J., Rufino di Aquileia, in A. Di Berardino (ed.), Nuovo dizionario patristico e di antichità cristiane, III, Genova-Milano 2008, 4612-4614.

Griffe E., La Gaule chrétienne à l'époque romaine, I, Paris 1964.

Grig L., Portraits, Pontiffs and the Christianization of Fourth-Century Rome, Papers of the British School at Rome 72 (2004), 203-230.

Grossi V., Giuliano d'Eclano, in A. Di Berardino (ed.), Nuovo dizionario patristico e di antichità cristiane, II, Genova-Milano 2007, 2309-2311.

Guire M., Nicetas of Remesiana, in New catholic Encyclopedia, X, Washington 2003.

Gutierrez L., El monaquismo romano y San Jerónimo, Communio 4 (1971), 49-78.

Gutierrez L., St Jerome and roman Monasticism. A historical Study on his spiritual Influence, Philippiniana sacra 10 (1975), 256-307.

Hammond C.P., The Last Ten Years of Rufinus' Life, Journal of Theological Studies 28 (1977), 372-429.

Happ M., Alte und neue Bilder vom Heiligen Martin. Brauchtum und Gebrauch seit dem 19. Jahrhundert, Köln 2006.

Healy R.B., The Carmina Natalicia of Paulinus of Nola, University of Western Australia 1975.

Heather P.J., Goths and Romans. 332-489, Oxford 1991, 193-224.

Heinzelmann M., Martin von Tours, in Reallexikon der Germanischen Altertumskunde, XIX, Berlin-New York 2001², 365-369.

Hellegouarc'h J., Le principat de Camille, Revue des Études Latines 49 (1971), 112-132. Hieronymus, Epistula 58 [Ad Paulinum presbyterum].

Hieronymus, Epistula 77, 10, CSEL [= Corpus Scriptorum Ecclesiasticorum Latinorum] $55,47$.

Hirschfeld O., Zur Camillus-Legende (1895), in IDEM, Kleine Schriften, Berlin 1913.

Hubaux J., Rome et Véies, recherches sur la chronologie légendaire du Moyen Age romain [= Bibliothèque de la Faculté de Philosophie et Lettres de l'Université de Liège, 145], Paris 1958.

Iustinianus, Codex 1. 2. 15. 1.

Iustinianus, Novellae 120. 1. 
Jones A.H.M., Martindale J.R., Morris J., The Prosopography of the Later Roman Empire, II, Cambridge 1980, 623 (Iovius 3).

Jülicher A., Iulianos 12, in Paulys Realencyclopädie der classischen Altertumswissenschaft, X/1, Stuttgart 1918, 19-22.

Jullian C., Ausone et Bordeaux. Études sur les derniers temps de la Gaule romaine, Bordeaux 1893.

Keay S. (ed.), Rome, Portus and the Mediterranean [= Archaeological Monographs of the British School at Rome, 21], London 2012.

Keay S., Boetto G. (ed.), Portus, Ostia and the Ports of the Roman Mediterranean, in E. Fentress et alii (ed.), XVII Conference of the Associazione Internazionale di Archeologia Classica (AIAC) (Rome 2008), Bollettino di Archeologia Online. Volume speciale, Roma 2011.

Keay S., Cascino R., Integrated approaches to the archaeology of Portus, in Porfyriou H., Genovese L. (ed.), Water shapes. Strategie di valorizzazione del patrimonio culturale legato all'acqua, Roma 2012, 131-148.

Keay S., Earl G., Felici F., Copeland P., Cascino R., Kay S., Triantafillou C., Interim report on an enigmatic new Trajanic building at Portus, Journal of Roman Archaeology 25 (2012), 486-512.

Keay S., Millett M., Paroli L., Strutt K. (ed.), Portus. An archaeological survey of the Port of Imperial Rome [= Archaeological Monographs of the British School at Rome, 15], London 2005.

Keay S., Paroli L. (ed.), Portus and its Hinterland: Recent Archaeological Research [= Archaeological Monographs of the British School at Rome, 18], London 2011.

Keay S., Portus and the Alexandrian Grain Trade Revisited, in E. Fentress et alii (ed.), XVII Conference of the Associazione Internazionale di Archeologia Classica (AIAC) (Rome 2008), Bollettino di Archeologia Online. Volume speciale, Roma 2011.

Kehne P., Gratian, in Reallexikon der Germanischen Altertumskunde, XII, Berlin-New York 1998², 598-601.

Kenney E.J., Clausen W.V., The Cambridge History of Classical Literature, Cambridge 1983.

La Fabbrica di San Pietro in Vaticano, Su questa pietra, catalogo della mostra, Città del Vaticano 2000.

Lamberigts M., Julian of Aeclanum: A Plea for a Good Creator, Augustiniana 38 (1988), $5-24$.

Lamberigts M., Recent Research into Pelagianism with Particular Emphasis on the Role of Julian of Aeclanum, Augustiniana 52 (2002), 175-198.

Laurence P., Ivresse et luxure féminines: les sources de Jérôme, Latomus 57 (1988), 885899.

Laurence P., Jérôme et l'ancilla Christi: servitude et liberté, Augustinianum 37 (1997), 411-429.

Laurence P., Jerôme et le nouveau modèle féminin [= Études Augustiniennes], Paris 1997.

Laurence P., Jérôme et les puellae: un vocabulaire connoté, Revista Agustiniana 38 (1977), 1039-1063. 
Laurence P., Jérôme, la culture grecques et les femmes, in B. Pouderon - J. Doré (ed.), Les apologistes chrétiens et la culture grecque [= Théologie historique, 105], Paris 1998, 315-331.

Laurence P., L'implication des femmes dans l'hérésie: le jugement de saint Jérôme, Revue des Études Augustiniennes 44 (1998), 241-267.

Laurence P., Les représentations de la domina chez Jérôme, Recherches de science religieuse 85 (1997), 41-55.

Laurence P., Rome et Jérôme: des amours contrariées, Revue bénédictine 107 (1997), 227-249.

Liber Pontificalis (Pars prior) 34, 16, in Th. Mommsen (ed.), Gestorum Pontificum Romanorum Liber Pontificalis [= Monumenta Germaniae Historica], Berolini 1898, 56-57.

Liddell H.G., Scott R., A Greek-English Lexicon. Revised and augmented throughout by Sir H.S. Jones, with the assistance of R. McKenzie, Oxford 1940.

Liebermann W.-L., Schmidt P.L., D. Magnus Ausonius, in R. Herzog (ed.), Restauration und Erneuerung. Die lateinische Literatur von 284 bis $374 n$. Chr. [= Handbuch der lateinischen Literatur der Antike, 5], München 1989, 268-308.

Lippold A., Ursinus und Damasus, Historia 14 (1965), 105-128.

Longosz S., Atellana w okresie patrystycznym, Vox Patrum 10 (1990), 273-291.

Longosz S., Czy Paweł Apostoł cytowat komediopisarza Menandra? Opinie Ojców Kościoła, Vox Patrum 9 (1989), 907-924.

Longosz S., Święty Augustyn a starożytny dramat teatralny, Vox Patrum 8 (1988), 369$-394,859-879$.

Longosz S., Widowiska teatralne zagrożeniem dla życia rodzinnego według św. Jana Chryzostoma, in J. Śrutwa (ed.), Chrześcijanie a życie publiczne w Cesarstwie Rzymskim III-IV w., Lublin 1988, 161-198.

Lössl J., Te Apulia genuit (c. Iul. imp. 6.18). Some notes on the Birthplace of Julian of Eclanum, Revue des études augustiniennes 44 (1998), 223-239.

Lössl J., Julian of Aeclanum on Pain, Journal of Early Christian Studies 10 (2002), 203$-243$.

Lössl J., Julian von Aeclanum. Studien zu seinem Leben, seinem Werk, seiner Lehre und ihrer Überlieferung, Leiden - Boston - Köln 2001.

Löx M., Monumenta sanctorum. Rom und Mailand als Zentren des frühen Christentums: Märtyrerkult und Kirchenbau unter den Bischöfen Damasus und Ambrosius, Wiesbaden 2013.

Luiselli B., Storia culturale dei rapporti tra mondo romano e mondo germanico, Roma 1992.

Mandouze A. (ed.), Storia dei santi e della santità cristiana, III: Vescovi e monaci riconosciuti dal popolo (314-604), Bergamo 1991.

Mara M.G., Ambrogio di Milano, Ambrosiaster e Niceta, in A. Di Berardino (ed.), Patrologia, III, Casale Monferrato 1978, 180-183.

Mara M.G., Niceta di Remesiana, A. Di Berardino (ed.), Dizionario patristico e di antichità cristiane, II, Casale Monferrato 1984, 2397-2398. 
Mara M.G., Niceta di Remesiana, A. Di Berardino (ed.), Nuovo dizionario patristico e di antichità cristiane, II, Genova - Milano 2007, 3489-3490.

Margarucci Italiani B.M., Il titolo di Pammachio, Santi Giovanni e Paolo, Roma 1985.

Maritano M., Delfino di Bordeaux, in A. Di Berardino (ed.), Nuovo dizionario patristico e di antichità cristiane, I, Genova-Milano 2006, 1354-1355.

Marrou H.I., La canonization de Julien d'Éclane, Historisches Jahrbuch 77 (1958), 434$-437$.

Martin P., L'idée de royauté à Rome, des origines au principat augustéen, I, Clermont Ferrand 1982, 360-382.

Marucchi O., Il primato di papa Damaso nella storia della sua famiglia, Roma 1905.

Matyszak Ph., Wrogowie Rzymu, Warszawa 2007.

McGrath A., Divine justice and divine equity in the controversy between Augustinus and Julian of Eclanum, The Downside Review 101 (1983), 312-319.

Meier M., Alarich und die Eroberung Roms im Jahr 410. Der Beginn der Völkerwanderung, in M. Meier (ed.), Sie schufen Europa. Historische Portraits von Konstantin bis Karl dem Großen, München 2007, 45-62.

Meier M., Patzold S., August 410 - Ein Kampf um Rom, Stuttgart $2010^{2}$.

Mensing R., Martin von Tours, Düsseldorf 2004.

Metz R., La consécration des vierges dans l'église romaine, Paris 1954.

Mirri L., Girolamo e l'ascesi femminile: dialogo spirituale e santità, in G. Matteuzzi (ed.), La relazione uomo-donna in una prospettiva spirituale. Un percorso storico [= Quaderni di San Sigismondo, 1], Bologna 2000, 13-23.

Mirri L., Il matrimonio in san Girolamo, Vox Patrum 16 (1996), 365-378.

Mirri L., La dolcezza nella lotta. Donne e ascesi secondo Girolamo [= Spiritualità occidentale], Bose - Magnano 1996.

Mirri L., La preghiera delle eremite del deserto, in La preghiera nel tardo antico. Dalle origini ad Agostino. XXVII Incontro di studiosi dell'antichità cristiana, Roma, 7-9 maggio 1998 [= Studia Ephemeridis Augustinianum, 66], Roma 1999, 591-624.

Mirri L., La teologia dello status monasticus in S. Girolamo, in Il monachesimo occidentale dalle origini alla Regula Magistri. XXVI Incontro di studiosi dell'antichità cristiana, Roma, 8-10 maggio 1997 [= Studia Ephemeridis Augustinianum, 62], Roma 1998, 267-289.

Mirri L., La verginità nel mistero di Maria in San Girolamo, in E.A. Livingstone (ed.), Studia Patristica, XXXIII, Leuven 1997, 325-344.

Mirri L., La vita ascetica femminile in san Girolamo [= diss. Pontificia Università San Tommaso d'Aquino], Roma 1992.

Mirri L., Rapporto tra continenza e matrimonio in S. Girolamo, Vox Patrum 19 (1999), 303-322.

Momigliano A., Secondo contributo alla storia degli studi classici [= Storia e letteratura, 77], Roma 1960.

Mommsen Th., Römische Forschungen, II, Berlin 1879.

Murphy F.X., Melania the Elder: a Biographical Note, Traditio 5 (1947), 59-77.

Murphy F.X., Rufinus od Aquileia, Washington D.C. 1945. 
Nautin P., Le premier échange épistulaire entre Jérôme et Damase: lettres réelles ou fictives?, Freiburger Zeitschrift für Philosophie und Theologie 30 (1983), 331-334.

Nazzaro A. (ed.), Giuliano d'Eclano e l'Hirpinia Christiana. Atti del Convegno Internazionale di Studi, Mirabella Eclano, 4-6 giugno 2003, Napoli 2004.

Nesi S., Il Comandante Nesi: un «Alcione» dalle ali spezzate, Milano 2004.

Nigg W., Martin de Tours: Chevalier du Christ, évêque thaumaturge, confesseur de la foi, Paris 1978.

Nowak P.J. (ed.), Sulpicjusz Sewer: Dialogi o życiu świętego Marcina, in P.J. Nowak (ed.), Sulpicjusz Sewer: Pisma o św. Marcinie z Tours, Kraków-Tyniec 1995, 109-190.

Nowak P.J. (ed.), Sulpicjusz Sewer: Listy, in P.J. Nowak (ed.), Sulpicjusz Sewer: Pisma o św. Marcinie z Tours [= źródła monastyczne, 8], Kraków-Tyniec 1995, 91-108.

Nowak P.J. (ed.), Sulpicjusz Sewer: Żywot świętego Marcina, in P.J. Nowak (ed.), Sulpicjusz Sewer: Pisma o św. Marcinie z Tours [= źródła monastyczne, 8], Kraków-Tyniec 1995, 49-90.

O'Flynn J.M., Generalissimos of the western Roman empire, University of Alberta Press 1983.

Ogilvie R.M. (ed.), A Commentary on Livy: Books 1-5, Oxford 1965, 669-670.

Ościłowski K., Ideał chrześcijanina i jego realizacja: studium pism św. Paulina $z$ Noli, Kraków 2009.

Oury G.-M., Saint Martin de Tours: L’homme au manteau partagé, Chambray-lès-Tours 1987.

Palladius, Historia Lausiaca 46.

Pasquato O., Spettacoli, in A. Di Berardino (ed.), Dizionario patristico e di antichità cristiane, II, Casale Monferrato 1984, 3279-3284.

Patin W.A., Niceta, Bischof von Remesiana als Schritsteller und Theologie, München 1909.

Patrologiae Latinae Supplementum 3, 189-202.

Paulinus Nolanus, Carmen 21, 398-403.

Paulinus Nolanus, Carmina, CSEL 30, 1-3, 7-329.

Paulinus Nolanus, Epistulae, CSEL 29, 92-97 (tr. italiana: G. Santaniello [ed.], Paolino di Nola. Le Lettere [= Strenae Nolanae. Collana di studi etici], Marigliano [NA] 1992.

Pennacchio M.C., Lesegesi patristica di Osea nei secoli IV-V. Teodoro di Mopsuestia, Teodoreto di Ciro, Cirillo di Alessandria, Girolamo, Giuliano di Eclano [= Studia Ephemeridis «Augustinianum», 81], Roma 2002.

Pietri Ch., Ghilardi M., Damaso, in A. Di Berardino (ed.), Nuovo dizionario patristico e di antichità cristiane, I, Genova-Milano 2006, 1325-1329.

Pietri Ch., Prosopographie de l'Italie chrétienne (313-604), I (A-K), Roma 1999, 1175$-1186$.

Pilara G., Alarico I, in A. Di Berardino (ed.), Nuovo dizionario patristico e di antichità cristiane, I, Genova - Milano 2006, 172-173.

Pippidi D.M., Niceta di Remesiana e le origini del cristianesimo daco-romano, Revue Historique du Sud-Est Européen 23 (1946), 99-117. 
Pismo Święte Starego i Nowego Testamentu. Najnowszy przekład z języków oryginalnych $z$ komentarzem. Opracował Zespół Biblistów Polskich z inicjatywy Towarzystwa świętego Pawła, s.l. et sine data, 2889.

Plutarchus, Vitae parallelae. Camillus (3).

Pollastri A., Melania senior, in A. Di Berardino (ed.), Nuovo dizionario patristico e di antichità cristiane, II, Genova-Milano 2007, 3187-3188.

Pollastri A., Pammachio, in A. Di Berardino (ed.), Nuovo dizionario patristico e di antichità cristiane, III, Genova-Milano 2008, 3789.

Polybius, Historiae 2, 18.

Prandi A., Ferrari G, The Basilica of Saints John and Paul on the Caelian Hill: After the Restorations and Archaeological Explorations Promoted by His Eminence, Francis Cardinal Spellman, Archbishop of New York and Cardinal Titular of the Basilica, Roma 1958.

Refoulé F., Julien d'Éclane, théologien et philosophe, Recherches de Science Religieuse 52 (1964), 42-84, 233-247.

Reutter U., Damasus, Bischof von Rom (366-384). Leben und Werk [= Studien und Texte zu Antike und Christentum, 55], Tübingen 2009.

Reynolds R.E., An early Medieval Mass Fantasy: The Correspondence of Pope Damasus and St Jerome on a Nicene Canon, in P. Linehan (ed.), Proceedings of the Seventh International Congress of Medieval Canon Law, Cambridge, 23-27 July 1984, Città del Vaticano 1988, 73-89.

Riggi C., La figura di Niceta di Remesiana secondo la biografia di Gennadio, Augustinianum 24 (1984), 189-200.

Riggi C., Niceta di Remesiana. Catechesi preparatorie al battesimo [= Collana di testi patristici, 53], Roma 1985.

Roberts W.E., Magnus Maximus: Portrait of a Usurper, University of South Carolina 1997.

Rücker N., Ausonius an Paulinus von Nola: Textgeschichte und literarische Form der Briefgedichte 21 und 22 des Decimus Magnus Ausonius, Göttingen 2012.

Rufinus, Prologus in Regulam Sancti Basilii.

Saddington D.B., The Educational Effect of Catechical Instruction in the Fourth Century A.D., Euphrosyne 5 (1972), 249-271.

san Pammachio Palladius, Historia Lausiaca 62, in G.J.M. Bartelink (ed.), Palladio. La storia Lausiaca [= Vite dei santi, 2], s.l. 1974, 268-269

Sanchez M., del Mar M., La visión de la mujer en San Jerónimo a través de su correspondencia, in E. Garrido González (ed.), La mujer en el mundo antiguo. Actas de las quintas jornadas de investigación interdisciplinaria organizada por el Seminario de Estudios de la Mujer. Universidad Autónoma de Madrid, Madrid 1986, 315-321.

Sciajno L. (ed.), Paolino di Nola. Il carme 15 (Natalicium IV) [= Studi sulla tardoantichità], Pisa-Roma 2008.

Seeck O., Alaricus 2, in Paulys Realencyclopädie der classischen Altertumswissenschaft, I/1, Stuttgart 1893, 1286-1291. 
Seeck O., Gratianus 3, in Paulys Realencyclopädie der classischen Altertumswissenschaft, VII/2, Stuttgart 1912, 1831-1839.

Selter B., Ausonius (Decimus Magnus Ausonius), in Ch. Walde (ed.), Die Rezeption der antiken Literatur. Kulturhistorisches Werklexikon [= Der Neue Pauly. Supplemente, 7], Stuttgart - Weimar 2010, 159-172.

Senjak Z., Niceta von Remesiana. Christliche Unterweisung und christliches Leben im spätantiken Dacien, Freiburg 1975.

Seppelt F.X., Geschichte der Päpste von den Anfängen bis zur Mittel des zwanzigsten Jahrhunderts, I: Die Entfaltung der päpstlichen Machtstellung im frühen Mittelalter. Von Gregor dem Grossen bis zur Mitte des elften Jahrhunderts, München $1955^{2}, 109-126$.

Sgarbossa M., I Santi e i Beati della Chiesa d'Occidente e d'Oriente, Milano 2000².

Sheperd M.H., The Liturgical Reform of Damasus, in P. Granfield - J.A. Jungmann, Kyriakon. Festschrift für Johannes Quasten, II, Münster 1970, 847-863.

Sivan H., Alaricus Rex. Legitimizing a Gothic King, in R. Corradini, M. Diesenberger, H. Reimitz (ed.), The Construction of Communities in the Early Middle Ages. Texts, Resources and Artefacts [= The Transformation of the Roman world. A Scientific Programme of the European Science Foundation, 12], Leiden 2003, 109-121.

Sivan H., Ausonius of Bordeaux. Genesis of a Gallic Aristocracy, London 1993.

Société des Bollandistes (ed.), Bibliotheca Hagiographica Latina, II, Bruxelles 1901, 823-830.

Sommer A.U., Das Ende der antiken Anthropologie als Bewährungsfall kontextualistischer Philosophiegeschichtsschreibung: Julian von Eclanum und Augustin von Hippo, Zeitschrift für Religions- und Geistesgeschichte 57/1 (2005), 1-28.

Soroceanu A., Niceta von Remisiana Seelsorge und Kirchenpolitik im spätantiken unteren Donauraum, Frankfurt a.M. 2013.

Stancliffe C., St. Martin and his hagiographer. History and miracle in Sulpicius Severus, Oxford 1983.

Starowieyski M., Wstęp, in P.J. Nowak (ed.), Sulpicjusz Sewer: Pisma o św. Marcinie $z$ Tours, Kraków-Tyniec 1995, 11-39.

Sulpicius Severus, Vita S. Martini, CSEL 1, 107-137.

Sulpicius Severus, Dialogi, CSEL 1, 152-216.

Sulpicius Severus, Epistula 1 [Ad Eusebium], CSEL 1, 138-141.

Świderkówna A. (ed.), Słownik pisarzy antycznych, Warszawa 2001.

Swoboda A., Pojęcie przyjaźni w listach św. Paulina z Noli i św. Sydoniusza Apolinarego: studium porównawcze [= Studia i Materiały - Papieski Wydział Teologiczny w Poznaniu, 17], Poznań 1995.

Täubler E., Camillus und Sulla, Klio 12 (1912), 219.

Taylor J., St. Basil the Great and Pope Damasus, Downside Review 91 (1973), 183-203, 261-274.

Testini P., Le catacombe e gli antichi cimiteri Cristiani in Roma [= Roma cristiana, 2], Bologna 1966, 101-105. 
Thonnard F.-J., Laristotélisme de Julien d'Éclane et de Saint Augustin, Revue des études augustiniennes 11 (1965), 296-304.

Titus Livius, Ab Urbe condita.

Traves P., Camillo, Marco Furio, in N.G. Lemprière Hammond, H.H. Scullard (ed.; ed. italiana: M. Carpitella), Dizionario di antichità classiche di Oxford, I, Roma 1981, 367-368.

Trout D. (ed.), Damasus of Rome: The Epigraphic Poetry, Oxford 2015.

Trovato S., Antieroe dai molti volti: Giuliano l'Apostata nel Medioevo bizantino, Udine 2014.

Turner C.H., De psalmodiae bono sive De utilitate credendi, Journal of Theological Studies 24 (1923), 225-252.

Turner C.H., De vigiliis (editio critica), Journal of Theological Studies 22 (1921), 305-320 .

Villegas Marín R., En polémica con Julián de Eclanum. Por una nueva lectura del Syllabus de gratia de Próspero de Aquitania, Augustinianum 43 (2003), 81-124.

Walsh G.C., Writings of Niceta of Remesiana [= The Fathers of the Church, 7), Washington 1949.

Walter U., Marcus Furius Camillus - die schattenhafte Lichtgestalt, in K.-J. Hölkeskamp, E. Stein-Hölkeskamp (ed.), Von Romulus zu Augustus. Große Gestalten der römischen Republik, München 2000, 58-68.

Wehrens H.G., Rom - Die christlichen Sakralbauten vom 4. bis zum 9. Jahrhundert - Ein Vademecum, Freiburg 2016, 189-194.

Wolfram H., Die Goten. Von den Anfängen bis zur Mitte des 6. Jahrhunderts; Entwurf einer historischen Ethnographie [= Frühe Völker], München 20014, 145-179.

Zeiller J., Un ancien évêque d'Illyricum, peut-être auteur du Te Deum, Saint Niceta de Remesiana, in Comptes-rendus de l'Académie des Inscriptions et Belles Lettres, Paris 1942, 356-369.

Zelzer M., Die Instructio ad competentes des Nicetas von Remesiana und die Salzburger Slawenmission, in Cristianesimo e specificità regionali nel Mediterraneo latino (sec. IV-VI). XXII Incontro di studiosi dellantichità cristiana. Roma, 6-8 maggio 1993 [= Studia Ephemeridis «Augustinianum», 46], Roma 1994, 321-327.

Zeppegno L., Mattonelli R., Le chiese di Roma [= Quest'Italia. Collana di storia, arte e folclore, 2], Roma 19854, 71-73. 